\title{
An Enhanced routing Technique to improve the Network Lifetime of Cognitive Sensor Network
}

V Jyothi ( $\square$ jyothinaik423@gmail.com )

JNTUA: Jawaharlal Nehru Technological University Anantapur

M.V. Subramanyam

Santhiram Engineering College

\section{Research Article}

Keywords: Channel allocation, Cognitive Event-driven Communication, sensor networks, WCETT, Energy consumption, Power dissipation

Posted Date: February 22nd, 2021

DOl: https://doi.org/10.21203/rs.3.rs-217361/v1

License: (c) (1) This work is licensed under a Creative Commons Attribution 4.0 International License. Read Full License 


\title{
An Enhanced routing Technique to improve the network lifetime of Cognitive Sensor Network
}

V Jyothi ${ }^{1}$ Dr.M.V. Subramanyam ${ }^{2}$

\begin{abstract}
In terms of using the technology of Cognitive Radio (CR), a Cognitive Sensor Network (CSN) is varied from the conventional Wireless Sensor Networks (WSNs). According to the interaction with the surrounding environment, the transmitter parameters can be modified in the sensor nodes of CSN adaptively. In CSNs, routing is one of the important components. Based on the capability of spectrum-aware, the schemes of routing of CSNs are district from other networks. The changeable spectrum resource dynamically should be understood by the routing scheme to establish a path of reliable forwarding by the adjustment of routing policy adaptively. In CSNs, reliable routing is an essential thing but still not a well-explored problem in CSNs. Packet drops due to spectrum unavailability and buffer overflows seriously affects the connectivity of the nodes. The whole network's lifetime and the data delivery rate are impacted by the prolonging packet drops. To increase the nodes' lifetime, the addressing of this drawback in the phase of routing should be done. Before the making of routing decisions, a new routing technique is proposed named as Drop factor based energy efficient routing technique (DFBEER) with the use of packet drop ratio and power dissipation metric of the spectrum links. With the total number of users in the routing path, the drop factor is computed. Power dissipation is calculated based on the transmitted data packets versus the amount of total consumed energy. This method reduces the drop ratio by avoiding the high drop factor nodes from being participating in the routing process. It always ensures that the data would be handled by the low dropping ratio nodes, thus the network's lifetime is improved.
\end{abstract}

Keywords: Channel allocation, Cognitive Event-driven Communication, sensor networks, WCETT, Energy consumption, Power dissipation.

\author{
V Jyothi \\ Research Scholar, Dept.of ECE, JNTUA, Ananthapuram, Andhra Pradesh, India. \\ jyothinaik423@gmail.com \\ Dr.M.V. Subramanyam \\ Principal, Dept.of ECE, Santhiram Engineering College, Nandyal, Andhra Pradesh, India. \\ santhiram775@gmail.com
}

\section{Introduction}

A vital networking paradigm has emerged as the Internet of Things (IoT) that allows communication among various physical objects. The improved devices with IoT including the applications in offices, households, and industrial devices soon would possess the capability of processing the data, sensing as well as transmitting the actions [1]. Owing to increment of enormous rates in the number of wireless devices, various challenges have to be faced by IoT things that include spectrum scarcity and energy. The energy-efficient and spectral techniques are required in IoT as these problems are originated with the usage of a wide range of sensor devices. For developing these spectral and energy-efficient methods, the below section includes the prime technologies.

For achieving the spectral efficiency, opportunistic spectrum sharing is the best solution [2]-[4]. Many connected devices can be supported by this solution for a wide range of applications. For opportunistic spectrum 
sharing, Cognitive Radio (CR) is the vital technology that can be allowed the unlicensed strategies (secondary users (SUs) for collaborating by the licensed network (primary network) [1], [2], [5]. Primary Users (Pus) represent the individuals who are subscribed for primary network. All non-contiguous spectrums can be utilized by CR sensor networks (CRSN) that make the spectrum more effective [6]. The issues of hardware and energy limitations can be imposed by CRSN that are adapted from WSN despite addressing the challenges of the dynamic spectrum. CR functionalities don't consider in the existing solutions for WSNs and challenges of the dynamic spectrum don't address. By not considering the hardware and energy challenges, the solutions are proposed for CRN. To find the solutions for CR and energy issues, new techniques are required to be found out. It's required to focus more on this work as CRSN has been gained attention from the research team. The current research works particularly on methods like connectivity, clustering, and path formation [7], [8].

In sensor networks, clustering is considered as one of the essential research fields that include the combination of the nodes in groups for management of the topology of a system. However, the stability of a system is improved. For wireless ad-hoc networks and WSNs, various reported works on clustering are considered [9], [10], [11], [12], and [13]. The whole network is categorized as self-organized clusters in the clustering in the existing schemes. Based on one of the cluster members known as cluster head $(\mathrm{CH})$, every cluster is controlled in the clustering process. Before the forwarding of $\mathrm{CH}$ towards next node within the path towards sink, the $\mathrm{CH}$ is acted as a cluster's central entity and the collected data from the cluster's other member nodes is aggregated. According to node's residual energy [14], the clustering schemes are selected CH for WSN. When CRSN networks have the availability of one common channel at least, the clustering methods can't be utilized for CRSN due to the two nodes are in a similar cluster. The assurance of stability doesn't be provided by this scheme alone owing to the occurrence of re-clustering with the dynamic radio environment.

In the automation in the industries and daily life processes, wireless sensor networks (WSNs) are playing a key role. WSNs have included numerous kinds of applications that include forest fire-detection, humidity monitoring, temperature monitoring, military target tracking and surveillance [15], agriculture [16], food health monitoring [17], and health applications [18]. WSNs have been made more attractive due to all of these applications so that they can be available everywhere. By using the vital components as WSNs [19], the developing smart cities are established their infrastructure.

Whenever the node1 of CRSN has some data for transmission, a three-step process has to be processed called a cognitive cycle [20] as PR nodes are considered as the priority nodes. To get aware of information about the condition of the current channel, channel sensing is performed firstly. The node of CRSN has decided to choose the sustainable channel or to tune for communication according to the results of channel sensing. Both unlicensed and licensed bands can be accessed by the nodes of CRSN and these bands will not provide interferences to the PR nodes harmfully. The node of CRSN has to be removed from the channel immediately to perform communication when in case of any PR node is appeared on a similar channel while making the communication of CRSN.

To create a static infrastructure-based wireless mesh network partly [21] or self-configuring ad-hoc network completely [22], the concept of cognitive radio can be implemented in general. To achieve an environment with complete spectrum awareness, the spectrum allotment map can be established and the neighborhood can be discovered fully in CRN which is based on infrastructure. This could be achieved with the accessing of availability of tools of upholding in the network that involves entities of central control. It will lead to the monitoring of allocation and accessing of the spectrum or geo-location databases. Such supporting tools are getting away in the assumed proposed network. However, the accessibility information of the spectrum is established at every cognitive node, in addition to the cooperation techniques which are needed within the medium of awareness of the local spectrum.

While considering the multiple QoS factors, the best rating can be achieved by a spectrum in a certain factor but similar performance couldn't be delivered. It's very important to choose the optimal spectrum as it can influence the QoS in allocation of secondary user. Hence, each spectrum's QoS metrics are being used for determining the best fit spectrum for allocation and sensing [23]. 
In the method of EACRP, the re-clustering is raised by selecting the $\mathrm{CH}$ nodes with the residual energy and distance of the nodes. Also, the border nodes of each cluster act as Gateway nodes for communicating with the neighbor cluster nodes. Beacon packets are used for GW communication. In this method, re-clustering is caused by considering the residual energy in the selection of $\mathrm{CH}$ due to the decreasing manner of the node's residual energy [45].

In the method of ESECR, the clustering has been used that is categorized the network into a different number of groups and allocated with $\mathrm{CH}$ which is made based on the nodes' residual energy. It will lead to the overhead of a network. Data packets are transmitted in an energy-efficient path. The network overhead is increased by the frequent Re-clustering within the Rotational $\mathrm{CH}$ selection method [46].

In previous works, it explains like ERCR, the routing is constructed based on high energy nodes. The energyefficient is constructed between the users based on energy availability. Although the residual energy in the routing mechanism is considered in this method, no proper mechanism for controlling and managing the energy consumption occurred due to retransmissions is present [47].

The energy efficiency is decreased in RITCB due to the assigning of nodes with the mechanism of sleep scheduling and the routing based on the nodes' idle time is considered. In this method, although the routing efficiency is improved, it fails to consider the energy efficiency of the network [48].

The paper proceeds as follows: Section 2 reviews various dropping and power based approaches in Cognitive Sensor Network. Section 3 explains the proposed approach for data dropping factor and power dissipation. Section 4 presents the simulation results and discussions. Section 5 is devoted to the conclusion of the paper.

\subsection{Contributions of this paper:}

Packet drops due to spectrum unavailability and buffer overflows seriously affects the connectivity of the nodes. The whole network's lifetime and the data delivery rate are impacted by the prolonging packet drops. The efficient selection of forwarder nodes helps to reduce the packet drop rate during data transmission. There are several energy-efficient route selection protocols that have been proposed in the past but none consider the packet drop factor and the methods to reduce it. The packet drops trigger repeated retransmissions until the dropped packet reaches the destination. These retransmissions affect the node energy consumption and reduce the network lifetime. Here we introduce a new enhanced routing technique that considers the node drop factor as the primary selection parameter. Also, the energy dissipation rate ensures the selection of optimal energy utilization nodes for the current round of communication. The drop factor is estimated against the average hop count between the communicating nodes. More hops lead to more packet drops. So the consideration of hop count between the nodes is important for fair selection. Also, the low energy dissipation of the nodes proves that the nodes participated well during data transmission in the past and utilized its energy optimally for data transmission. So, the consideration of drop factor and energy dissipation rate helps to increase the lifetime of the nodes than the previously proposed lifetime improving protocols.

\section{Advantages of the proposed method:}

- The parameters drop factor and energy dissipation rate are very lightweight to compute. Thus no additional overhead to the network.

- The drop factor is estimated against the average hop between the nodes. The minimum hops between the communicating nodes consume less energy.

- The energy dissipation parameter helps to select the nodes with optimal energy utilization in the past.

- The number of retransmission due to link failure and other possible reasons is completely controlled and the data delivery rate is increased considerably.

- The proposed protocol gives less overhead to the network and improves the overall network lifetime.

\section{Highlights}


- We proposed an enhanced routing technique Cognitive sensor networks that improve the lifetime of the network.

- The proposed routing technique selects forwarder nodes based on the values estimated using the drop factor and energy dissipation rate of the nodes.

- The drop factor selection parameter reduces the packet drop rate by avoiding the high drop factor nodes from being participating in the routing process.

- The consideration of the energy dissipation parameter ensures the optimized energy utilization among the sensor nodes.

- The reduced packet drop rate reduces the overhead of the network as the packets are delivered without any interruption in the routing path.

- The proposed routing technique is light-weight and efficient compared to traditional energy-efficient routing protocols.

\section{Literature Survey}

To deal with the scarcity of energy, energy harvesting has been included for CR networks recently. Energy harvesting is introduced by authors in [24]. For improving the throughput under the conditions of collision and energy causality for the accessing of the spectrum, optimal harvesting is presented in CR networks. For spectrum sharing as well as wireless energy harvesting, a cooperative mechanism is proposed in 5G networks by authors in [25].

Based on the constraints on energy harvesting and data rate, the formulation of an optimization problem is done to increase both SUs and Pus' throughput. For energy harvesting, a method of channel selection is proposed in CR networks for attaining the throughput of SUs with the maximum amount under the conditions of fading channel and energy neutrality which was mentioned in [26].

According to the sensor nodes' distance from the sink and event, candidate sensor nodes on behalf of $\mathrm{CH}$ are demonstrated primarily. By relying on available channels, node degree, in addition to the distance out of the sink within the respective neighborhood, $\mathrm{CH}$ is chosen out of candidate nodes. The strategies of $\mathrm{CH}$ selection are described in [27] and [28] that choose $\mathrm{CH}$ according to the weight. From the cluster's average energy nodes and sensor nodes' residual energy, the weight is computed. In [29], a scheme of energy-efficient $\mathrm{CH}$ has been proposed similarly where $\mathrm{CH}$ is chosen from the cluster's inner area nodes. For clustering in WSNs, a binary qualitative connectivity data and quantitative location data is exploited by authors in [30]. In the selection of $\mathrm{CH}$, another different class is restricted to the combination of various factors like distance, energy, and signal strength.

The requirement of bandwidth, optimal routing activities, and energy consumption are achieved with the existing schemes that involve clustering and maintenance of network topology. The main objectives of the proposed schemes in cognitive radio ad-hoc networks [31] and [32] are involved the maximization of spectrum utilization and minimization of energy consumption.

By CRSNs, they can't be used. In recent works [33], [34], and [35], a few types of methods are also improved for CRSNs. Still, it's required to determine more efficient techniques that can solve both the issues of the spectrum and energy scarcity jointly. It will be helped to achieve further evolution in IoT things.

For cognitive-radio-based WSNs, a technique of an energy-efficient and learning inspired channel decision is performed in [36]. According to the previous information relevant to the energy efficiency and energyconsumption rate obtained by the CRSN, any available channel can be accessed by sensor nodes in CRSN. For multi-channel CRSN, the energy-efficient and lifetime of a network are improved which is mentioned in [37]. In addition to the assignment of energy-aware channel on behalf of nodes of a sensor, the adoption of packet size is used by the scheme.

To increase the usage of scarce spectrum, the technique of CR is helped. While restricting the harmful interference, the spectrum opportunities or white spaces are utilized in a network of CR nodes. To create the opportunities, the CR node is a better chance which is provided by the intelligent decision. By relying on the CR 
nodes with a total number in a network, the utilization of an intelligent channel is mentioned by the authors in [38]. The required performance can be provided by the probability-based channel selection when the traffic of CR is low in the network. In the selection of the required channels, better results are provided by sensing based channel selection for high CR traffic. For CR based networks, the essential function of the techniques of channel prediction is highlighted in [39]. To determine the channels' sustainability for CR nodes, various methods have been considered. The switching of CR nodes to the channels that provide longer idle slots based on the coordination of a network [40] using these techniques. The prediction based schemes are discussed by authors in [41] for the activity of PR users. By decreasing the number of switching channels, the outcome of the CR node can be enhanced. For the prediction of the forthcoming PR activities and to decrease the harmful interference effectively [42], the histories of the past channel can be used intelligently by the CR nodes.

Based on the dissimilar delay-based as well as energy-based levels, the routing protocol of CRN is classified in [43]. To determine the route with the smallest weight, the algorithm of Dijkstra is utilized at each level. To gather and communicate the information of channel availability with the nodes of SU, the algorithm is dependent on a central controller or base station.

The network's lifetime in addition to energy consumption of nodes is considered as metrics by the authors in [44]. Based on the existing several packets within the queue of the nodes in addition to the usual energy usage per packet, the consumption of energy is computed. The implementation of the MAC protocol is not particularly mentioned.

Ram Narayan Yadav et.al is implemented as an approach of energy-effective as well as distributive event-driven cluster-based routing for making the robust CRSN to PU actions. The intra-cluster aggregation is used by the spectrum aware clustered structure based on $\mathrm{CHs}$ in addition to effective inter-cluster relaying. Under the constraint of spectrum awareness, the clustering is utilized to reduce the intra-cluster distances in addition to the intra-cluster communication power. Depending upon the available channel, residual energy, in addition to the distance towards the sink nodes, gateway nodes for connectivity of inter-cluster and $\mathrm{CHs}$ for each cluster are chosen [45].

Saleem Aslam et al. is presented as an energy and spectrum efficient technique on behalf of the cognitive radio sensor networks (CRSN) for IoT. Using sensor nodes, the harvesting energy and spectrum can be accessed through the sources of ambient RF. An energy management approach is presented that involves a cluster head selection method and an energy-aware mode switching technique. For the allocation of the superior channel quality towards the sensor nodes concerning reliability as well as stability, a channel management approach is proposed additionally that is effective than the previous approaches based on the simulation results [46].

Rita Ahmed Abu Diab et.al has been developed a routing protocol of devising a lightweight cognitive radiobased algorithm to allow the IoT devices to communicate with one another and to act as SUs based on the multihop connections above an approved radio channel. By reducing the communication overhead and number of hops, the protocol is tried to balance the consumption of energy among SUs, achieve the highest possible pernode capacity, and decrease the energy usage. The following contributions are combined with the proposed routing scheme [47].

Syed Hashim Raza Bukhari et al. (2018) has been presented two methods known as residual idle time aware intelligent channel bonding with interference prevention (RITCB-IP) in addition to residual idle time aware intelligent channel bonding (RITCB) on behalf of the cognitive radio sensor networks. Four methods are compared such as sample width algorithm, the scheme of the primary radio user activity aware channel bonding, cognitive radio network across the white spaces with AGILE. By using the RITCB-IP and RITCB, the life-space of cognitive radio sensor node is improved and the dangerous interferences are reduced based on the simulation results [48].

In this paper, a novel meticulous progression based GA method is devised for QoS adequacy latitude aware routing for Cognitive Wireless Mesh Networks. In the past research studies, spectrum sensing strategies were focused only on the optimality data transmission and they couldn't be supported for the desired frequency in Cognitive Wireless Mesh Networks. To overcome all these limitations, the proposed technique has been utilized 
through Meticulous Progressions based Genetic Algorithm. Because of the restrictions in the resources of cognitive wireless mesh network and computational complexities in GA [49], very limited solutions were selected for the optimality strategies of QoS for GA.

\section{Proposed work}

The connection among the nodes is affected by the packet drops that can be occurred due to the buffer overflows and the unavailability of the spectrum. The delivery rate of data and the network's lifetime are impacted by the prolonging of packet drops. Power is the vital factor to keep the nodes alive. Power is consumed for every data packet transmission. Nodes consume power for every data packets based on different network conditions such as distance, radio range, initial power...etc. Power wastage is inevitable in wireless networks due to various reasons like unexpected traffic, obstacles. High power nodes must be chosen for transmission of the data for longer communication. With the intention to increase nodes' lifetime, the disadvantage in the phase of routing must be detected. Before considering the decisions for routing, a new routing method is proposed with the use of the packet drop ratio and power dissipation metric of the spectrum links. The calculation of the drop factor is made with the involved number of users in the path of routing. Power dissipation is estimated based on the difference of total number of data packets transmitted and total power consumed. By restricting the high drop factor \& high power dissipating nodes to have participated in the routing process, the drop ratio is reduced by implementing this technique. Based on the nodes with low dropping ratio and low power dissipation, the dealing of data is ensured always that leads to the improvement in the network's lifetime.

For the cognitive sensor network, the protocol of WCETT is used in the work.

A randomness feature to the existing protocol is induced by the WCETT protocol which is extended with a drop factor. The decision of either forwarding or dropping of the packet of RREQ towards the destination is taken by every router or intermediary nodes amid source nodes as well as destination nodes during route discovery. The drop factor is computed by each node before making the forwarding of an RREQ packet. Here, the drop factor is a parameter of the inverse of the traversed number of hop counts by the packet of RREQ. In general, the value of the drop factor is between 0 to 1 . A random number from 0 to 1 is produced by a node. The packet of RREQ is forwarded by the node when the random number is more than the drop factor. The packet of RREQ is dropped otherwise. With the source node, a process of new route discovery is necessarily not resulted from the dropping of packets of RREQ. The multiple packets of RREQ through the neighbors result due to the fact of the original broadcasting with the help of a source node. Within several packets of RREQ traversing a network in the destination search, this diffusing wave results. The best route can be determined by a single packet of RREQ owing to the redundancy of the major proportion of the packets. The diffusing of a number of these packets away from the destination in different directions shall timeout eventually. The important intention of the protocol is to decrease the transmission of redundant packets of RREQ. These redundant packets of RREQ can be dropped as much as possible. With the increased number of hops, the value of drop policy becomes lesser and it is conservative. The survival of RREQ packets with chances are increased due to the packets of RREQ is nearer to the destination node. The process of the first phase of the route discovery and determination of the destination node is accomplished as soon as possible. From the destination node, the transmission of an RREP packet to the source node can be done. A proportion of RREQ packets is reduced by the dropping of redundant RREQ packets in the proposed protocol. These packets don't reach the destination node never that decreases the congestion in a network. In this proposed protocol, the ratio of received packets using nodes towards various packets forwarded through nodes is termed as throughput is higher. To improve the network's lifetime, this reduced congestion is assisted. Fig 1 represent the block diagram of proposed system. 


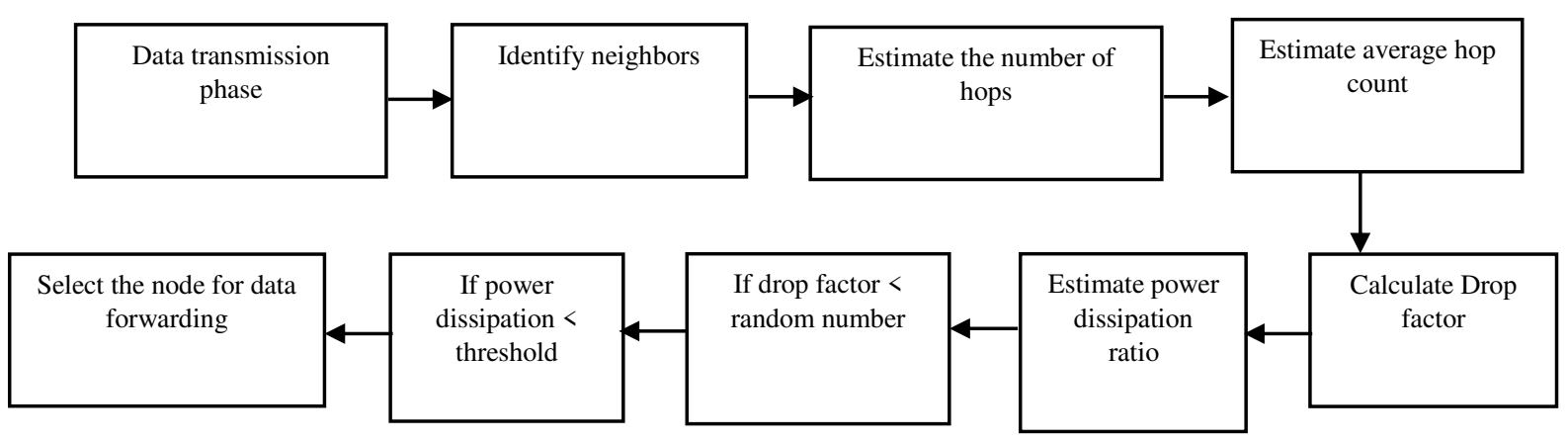

Fig 1: Block diagram of proposed system

\subsection{Routing metric}

In multi-hop CRNs, the routing metric is utilized that should be reflected in the activities of PU, quality of links, the availability of bands, and SUs with QoS requirements. To determine the routing paths with high-throughput in multi-hop and multi-radio CRAHN, the WCETT metric is exploited in this paper. According to the quality and combining the weights of links, the weights to each link are assigned by this metric. The calculation of WCETT is displayed in the below section.

\subsection{Weighted Cumulative Expected Transmission Time (WCETT)}

The computation of the path metric is suggested by the WCETT metric which represents the extra time of ETT parameter [74]. The metric value is more when compared to addition of individual links with parameter values that belong to this path. When the same channel is used, the interference of concatenated links may be done with each other by not considering the summation. In the end-to-end routing path, WCETT aims to decrease the interference of intra-flow with the minimization of the number of nodes in a similar channel. Let's consider $\mathrm{N}$ as the system's overall number of channels, overall hops the sum of transmission times upon a channel $\mathrm{j}, 1 \leq \mathrm{j} \leq \mathrm{N}$, is considered as mentioned below:

$$
X_{j}=\sum_{j \text { is used on link } l}^{n} \text { ETT }
$$

A weighted average between the sum of all ETTs and the maximum value of $\mathrm{Xj}$ is proposed as the domination of throughput of a total path will be done based on the bottleneck channel that includes the largest $\mathrm{Xj}$.

Based on the below formula, it results.

$$
W C E T T=(1-\beta) \sum_{l \in p} \operatorname{ETT}(l)+\beta \times \max _{1 \leq j \leq N} X_{j}
$$

Due to the paths that include lower weights with more channel diversity, the term of $\max 1 \leq j \leq N X j$ is captured the interference of intra-flow explicitly and $\beta$ is the tunable parameter $0 \leq \beta \leq 1$. A tradeoff amid channel diversity of a chosen route (second term) in addition to the path latency (first term) can be viewed in (2). The effect of $\beta$ upon the performance of routing within the WSNs of multi-radio multi-channel multi-hop is studied thoroughly by the authors in WCETT and the effect is shown in the throughput. To balance the path latency and the channel diversity, $\beta=0.5$ is selected as similar to the proposed model.

\subsection{Algorithm}

$\mathrm{N}$ - Total number of nodes;

$\mathrm{nn}$ - number of nodes;

DEST - destination node 
spec - spectrum;

Df - drop factor; $P D_{n}-$ Power dissipation metric of node $\mathrm{n}$

$N O P_{n}=$ No. of packets transmitted by node $\mathrm{n}$

$P_{\text {thresh }}=$ Power threshold value for every data transmission

Avg_hc - Average hop count;

Rand - random value b/w 0 and 1

Sel_node[] - selected node

WCETT - Weighted Cumulative Expected Transmission Time;

Val(WCETT) - WCETT value

Seq_no - Sequence number; LT - local table

For the node $n$, where $n \exists N$

Set $P D_{n}=0$

Determine the Drop factor DF(n)

Calculate the average hop count Avg_hc(n)

Broadcast control packets

Update the neighbour table

Determine the total neighbours nn from neighbour table

Estimate Drop factor $\operatorname{DF}(n)=[1 / \operatorname{Avg}$ hc $(n)+1]$

Estimate $P D_{n}=A P_{n}-\left[N O P_{n} * P_{\text {thresh }}\right]$

Compute rand(n) [any random number between 0,1 ]

If $\left(\operatorname{rand}(\mathrm{n})>\operatorname{Df}(\mathrm{n}) \& \& P D_{n}<\right.$ treshold $)$

Choose the node $\mathrm{n}$ for assessment of WCETT

Sel_node $[\mathrm{i}]=n \quad$ Where $\mathrm{i}=0$ to $\mathrm{N}$

End If

End For

For the node $n$, where $n \exists N$

PU broadcast RREQ to DEST

Compute the chosen node's WCETT (Sel_node[n])

Node broadcast RREQ packets

Nodes receiving the RREQ packets

Check the sequence number of RREQ packets

If (Seq_no[RREQ] == new) 
Store the value in Local table LT

$\mathrm{LT}[\mathrm{i}]=\operatorname{val}(\mathrm{WCETT})$

Else

(Seq_no[RREQ] is Exist)

Compare the value with the existing value

If $(\operatorname{val}($ WCETT) < val(WCETT)-1)

$\mathrm{LT}[\mathrm{i}]=\operatorname{val}(\mathrm{WCETT})$

End IF

End If

End for

For Data transmission

For the node $n$, where $n \exists N$

Check for the High value forwarder nodes

If $\mathrm{LT}[\mathrm{n}]<\mathrm{LT}[\mathrm{n}+1]$

$\mathrm{FN}=\mathrm{LT}[\mathrm{n}]$

Else

$\mathrm{FN}=\mathrm{LT}[\mathrm{n}+1]$

End If

End for

End Data transmission

\section{Results and discussion}

Based on the extensive simulations with the help of NS-2 network simulator, the proposed scheme DFBEER's performance is analyzed in this paper. The results with four existing competitive schemes like EACRP, ESECR, ERCR, and RITCB are compared to prove the effectiveness of DFBEER. To route in Mobile Ad-Hoc Networks, the method using the principle of routing protocol of on-demand distance vector (AODV) is designed. By considering the energy in routing, the cognitive feature within AODV is combined, as well as assigning of joint node-channel is performed. In the method of ERCR, the routing and energy efficiency are constructed between the users depending upon high energy nodes and energy availability respectively. $\mathrm{CH}$ nodes are chosen depending upon the remaining energy in addition to the distance of nodes in EACRP and routing processes in RITCB. The clustering of a network is utilized in ESECR. However, all of these methods ERCR, RITCB, EACRP, and ESECR are compared. The considered schemes' results are compared concerning end-to-end packet delay, energy consumption, routing overhead, in addition to throughput. The parameters for simulation are given in Table 1and these are summarized that are used in various experiments. Over an area of $1000 \times 500$ $\mathrm{m} 2,1000$ stationary nodes are distributed randomly that includes in the simulation setup. A transmission range of $250 \mathrm{~m}$ using an interference range of $250 \mathrm{~m}$ is reached with each node by connecting to other nodes. By sending 1024-byte packets at a rate of $0.1 \mathrm{~ms}$, the traffic sources are completely having a constant bit rate (CBR) and variable bit rate (VBR). Based on the total energy of $100 \mathrm{~J}$, each node is initiated.

Table 1: Simulation table 


\begin{tabular}{|l|l|}
\hline PARAMETER & VALUE \\
\hline Application Traffic & CBR, VBR \\
\hline Transmission rate & 1024 bytes/0.1 ms \\
\hline Radio range & $250 \mathrm{~m}$ \\
\hline Packet size & 1024 byes \\
\hline Maximum speed & $50 \mathrm{~m} / \mathrm{s}$ \\
\hline Simulation time & $50 \mathrm{secs}$ \\
\hline Number of nodes & 1000 \\
\hline Initial Energy & 100 joules \\
\hline Area & $1000 x 500$ \\
\hline Routing Protocol & WCETT \\
\hline Number of Interfaces & 3 \\
\hline Routing Methods & $\begin{array}{l}\text { DFBEER, ESECR, ERCR, RITCB } \\
\text { EACRP, }\end{array}$ \\
\hline
\end{tabular}

In the area of $1000 \times 500$ square meters, the nodes are deployed randomly for the simulations. For sensor nodes, the maximum transmission range is 200 meters. For the usage of both sensor nodes and Pus in the area, multiple channels are presented. One of the licensed channels is chosen by Pus for communication.

For simulation experiments with five different sets, the performance results are introduced in this section. Under the varied traffic loads of the SU network, the performance of DFBEER is studied by the first set. By varying the number of traffic flow, the realization of variation of traffic load is considered even though VBR and CBR sources are used with a fixed traffic rate. Up to network's one-third of the SUs, the number of traffic flows is varied simultaneously and are acted utilizing traffic generators that are 10 traffic flows. On the performance of DFBEER, the impact of improving the PU activity's likelihood is investigated in the second series of observations. The effect of a value of the energy threshold upon the performance parameters of DFBEER, the third series of observations is studied. Effect of transmission range on the DFBEER performance is investigated in the fourth set of experiments regarding the percentage overhead. On an application scenario of cognitive practical, the DFBEER's performance is introduced in the fifth set. From at least 50 simulation runs, the results are considered in every set of experiments and average standards of the overall runs are represented. The varying node location is done in a random way at each run. For all performance results of DFBEER, the representation of the sample runs with standard deviation by error bars is provided. 


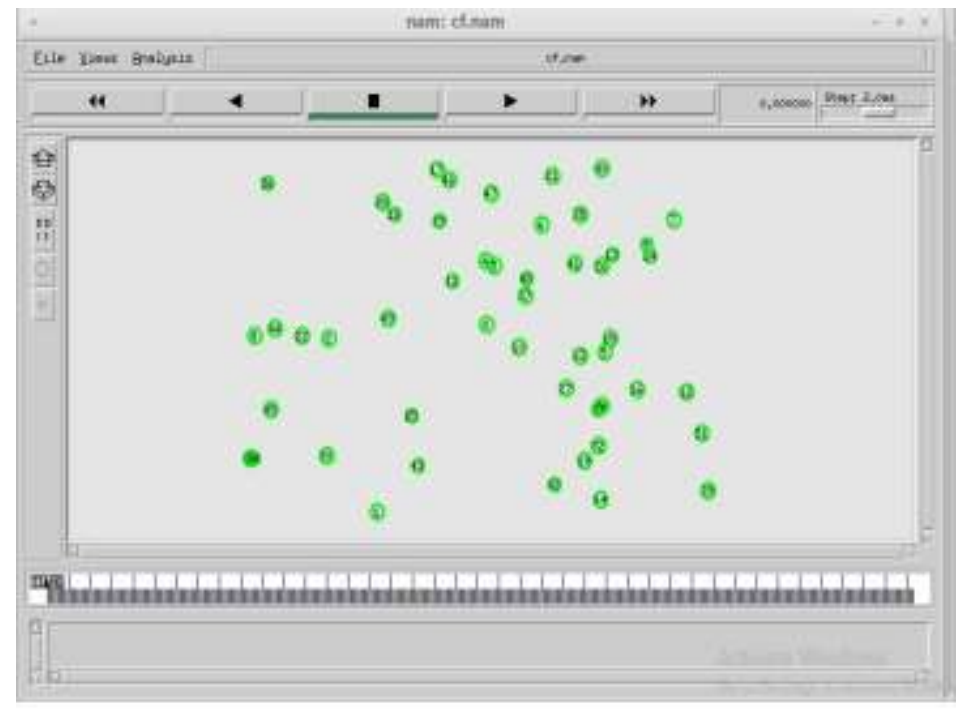

Fig 2: Network Deployment

In fig 2, it represents the Network deployment. Where the deployment of the overall nodes is done initially.

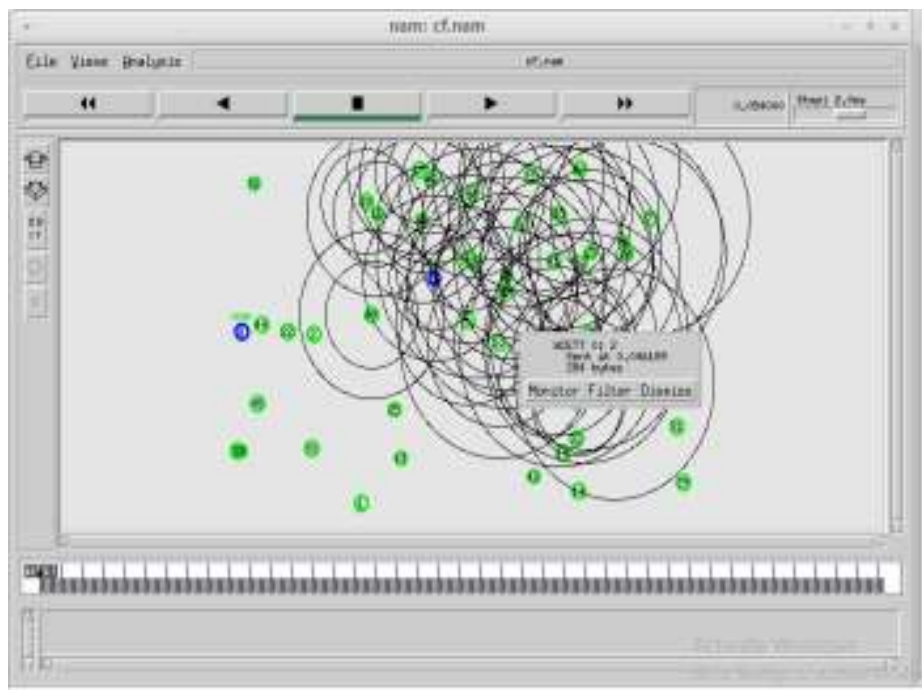

Fig 3: Broadcasting in the network

In fig 3, broadcasting process in the network, once the nodes deployment is completed in the network, is represented. Checks whether the node is present or not in its range. 


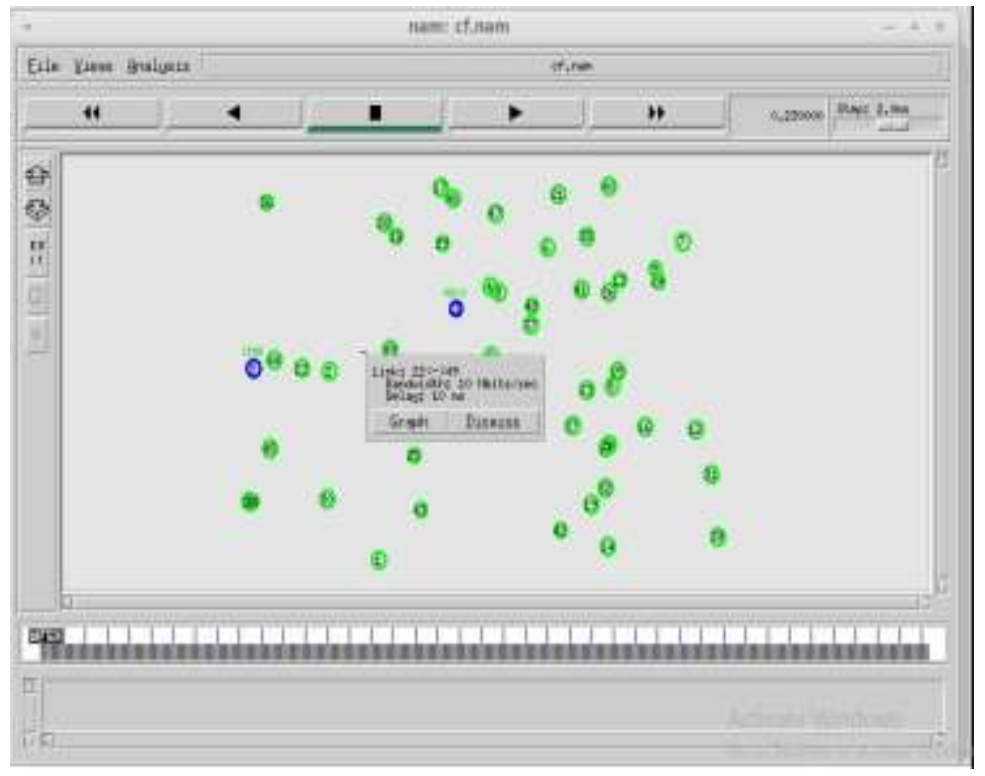

Fig 4: Resource availability in the links

Fig 4 shows that resource availability in the communication links and approximately estimated delay is $10 \mathrm{~ms}$.

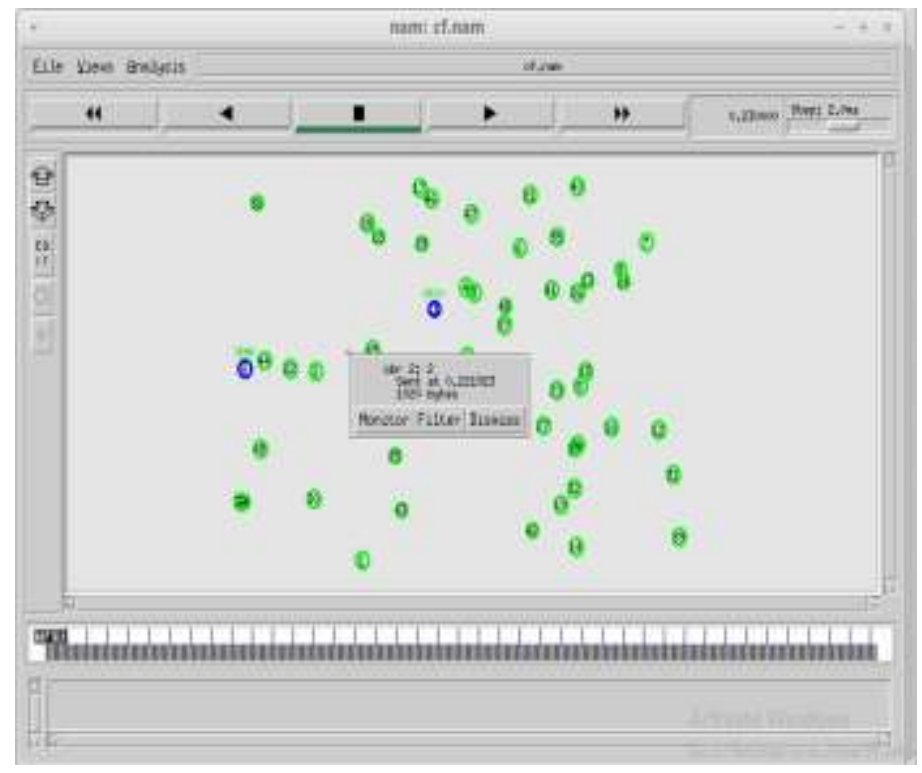

Fig 5: Data transmission at the rate of 1020 bytes between PAIR 1

Fig 5 represents the data transmission at the rate of 1020 bytes between PAIR1. The PU1 is transmitting data towards the target node using a data rate of 1020 bytes. 


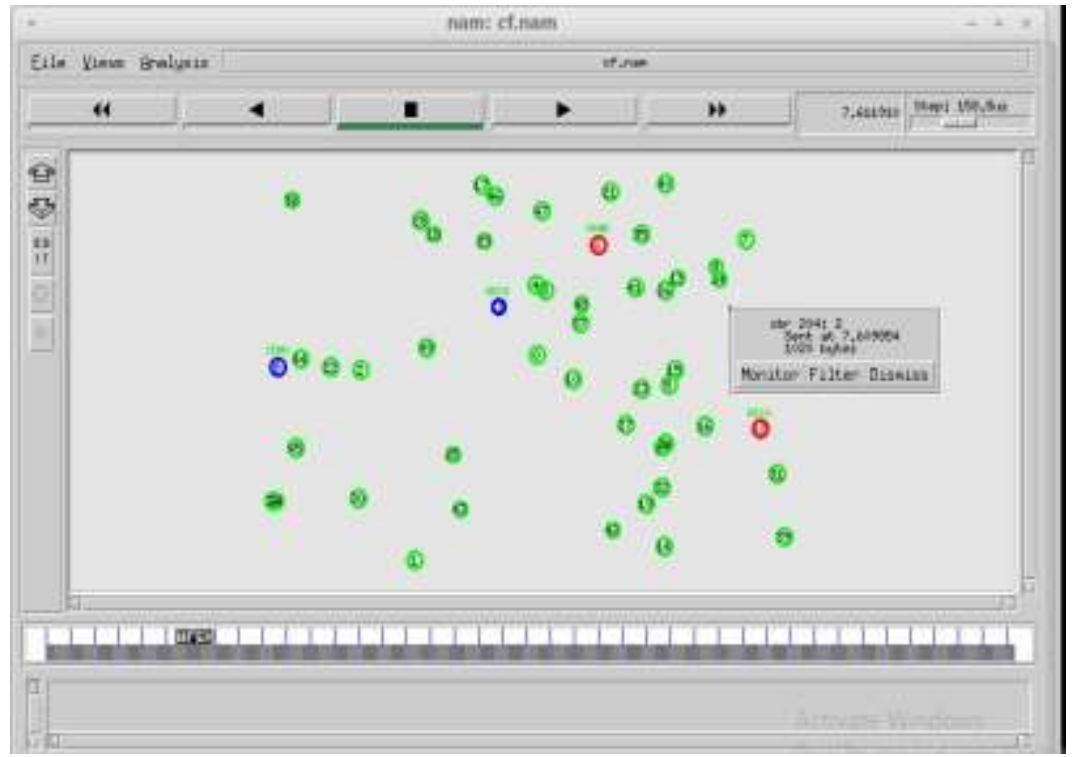

Fig 6: Data transmission at the rate of 1020 bytes between PAIR 2

Fig 6 shows that data transmission at the rate of 1020 bytes between PAIR2. The PU2 is transmitting data towards the target node using a data rate of 1020 bytes.

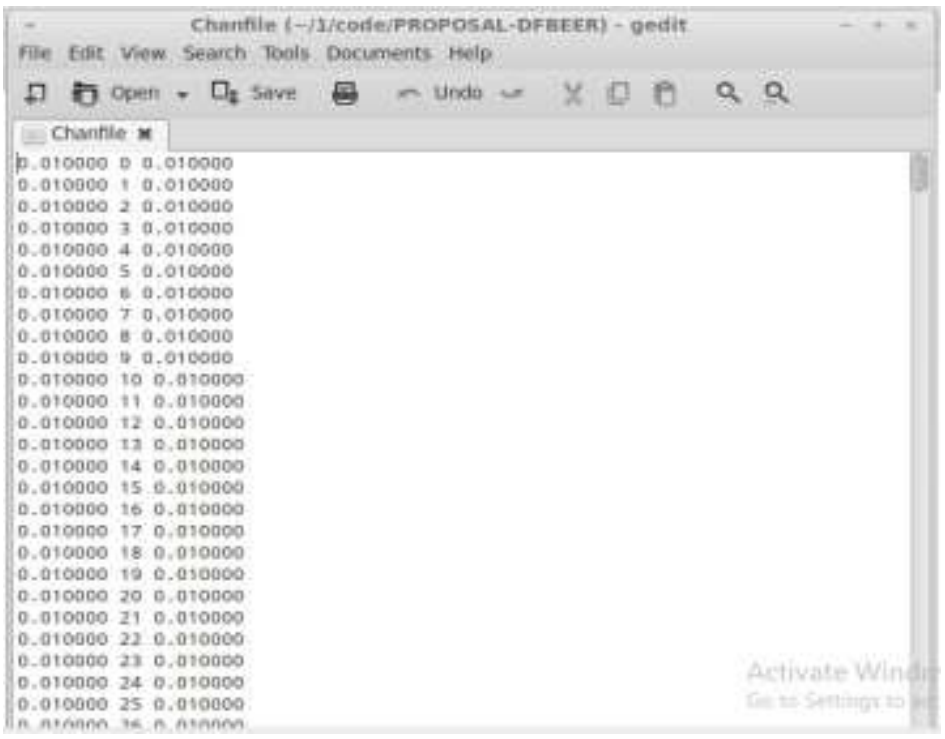

Fig 7: Channel utilization of the nodes during run time (time, node id, utilization)

In fig 7, it represents the Channel File during run time, here at every second of network performance is observed. 


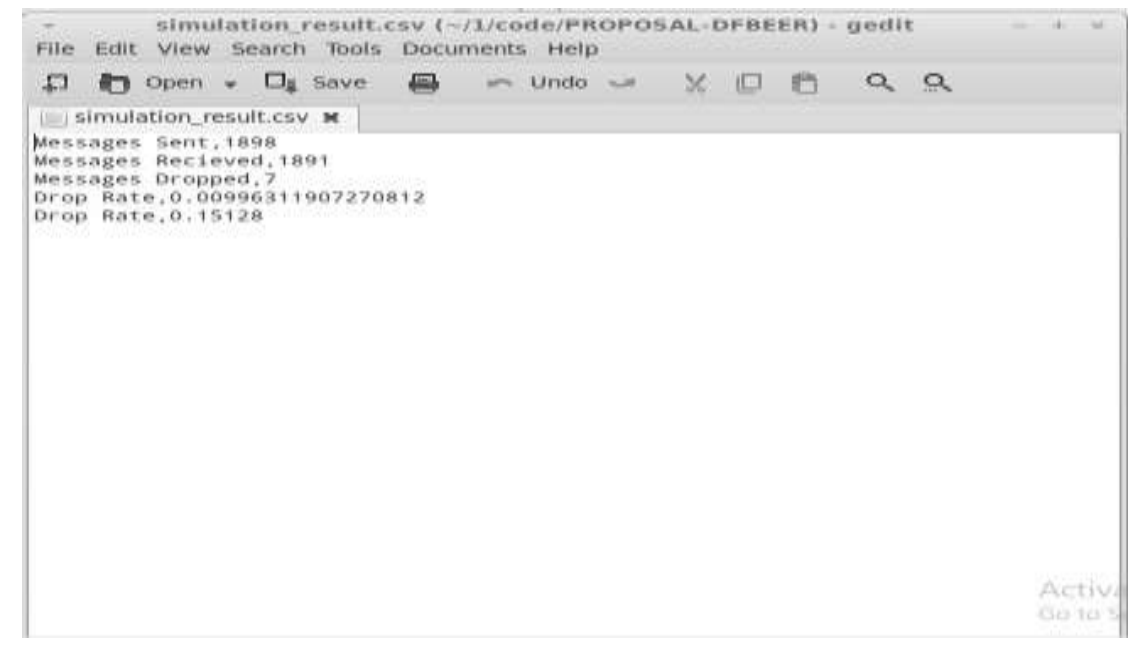

Fig 8: Data transmission report after the simulation ends

In fig 8 , the simulation results are shown. The overall performance is calculated and displayed.

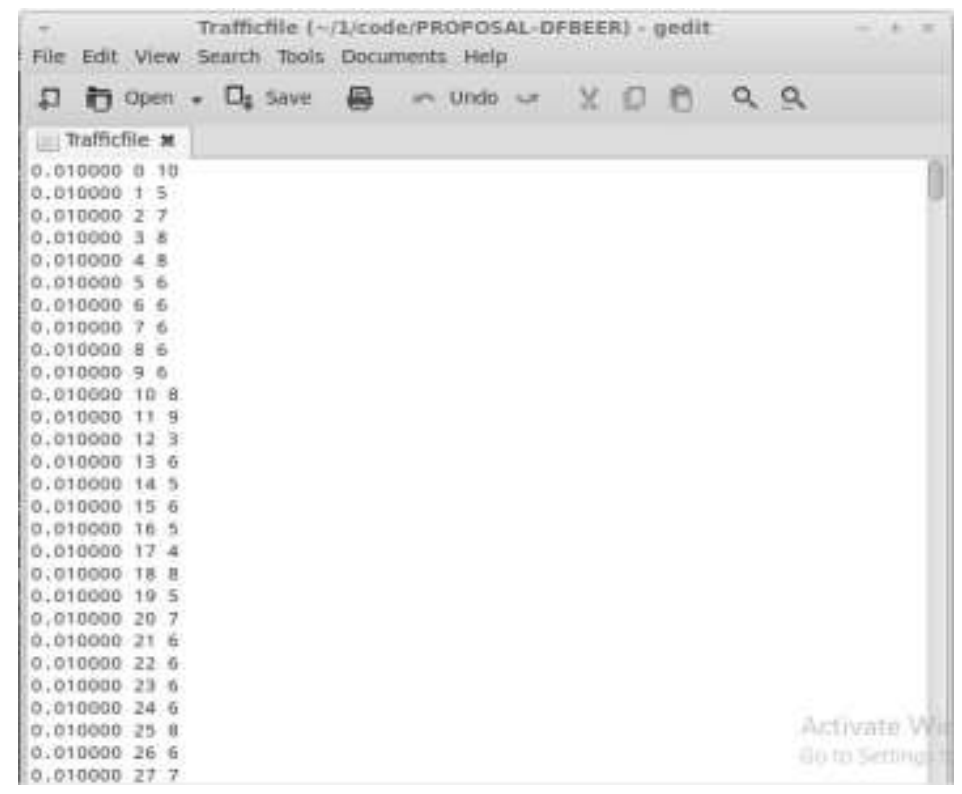

Fig 9: Traffic report of the nodes during run time (time, node id, traffic count)

In fig 9, the Traffic report for the nodes during run time in the network is represented. Here time, node id, traffic count generated in the traffic file.

\section{Performance evaluation:}

This section compares the network performance achieved by proposed with EACRP, ESECR, ERCR, and RITCB techniques in terms of the following performance metrics:

Table2: Comparison of different protocols in a delay performance

\begin{tabular}{|l|l|l|l|l|l|}
\hline Protocols & 200 & 400 & 600 & 800 & 1000 \\
\hline EACRP & 0.193 & 0.202 & 0.246 & 0.283 & 0.312 \\
\hline ESECR & 0.209 & 0.221 & 0.268 & 0.301 & 0.328 \\
\hline ERCR & 0.139 & 0.156 & 0.193 & 0.239 & 0.288 \\
\hline RITCB & 0.176 & 0.189 & 0.212 & 0.256 & 0.299 \\
\hline PROPOSED & 0.086 & 0.108 & 0.178 & 0.21 & 0.259 \\
\hline
\end{tabular}




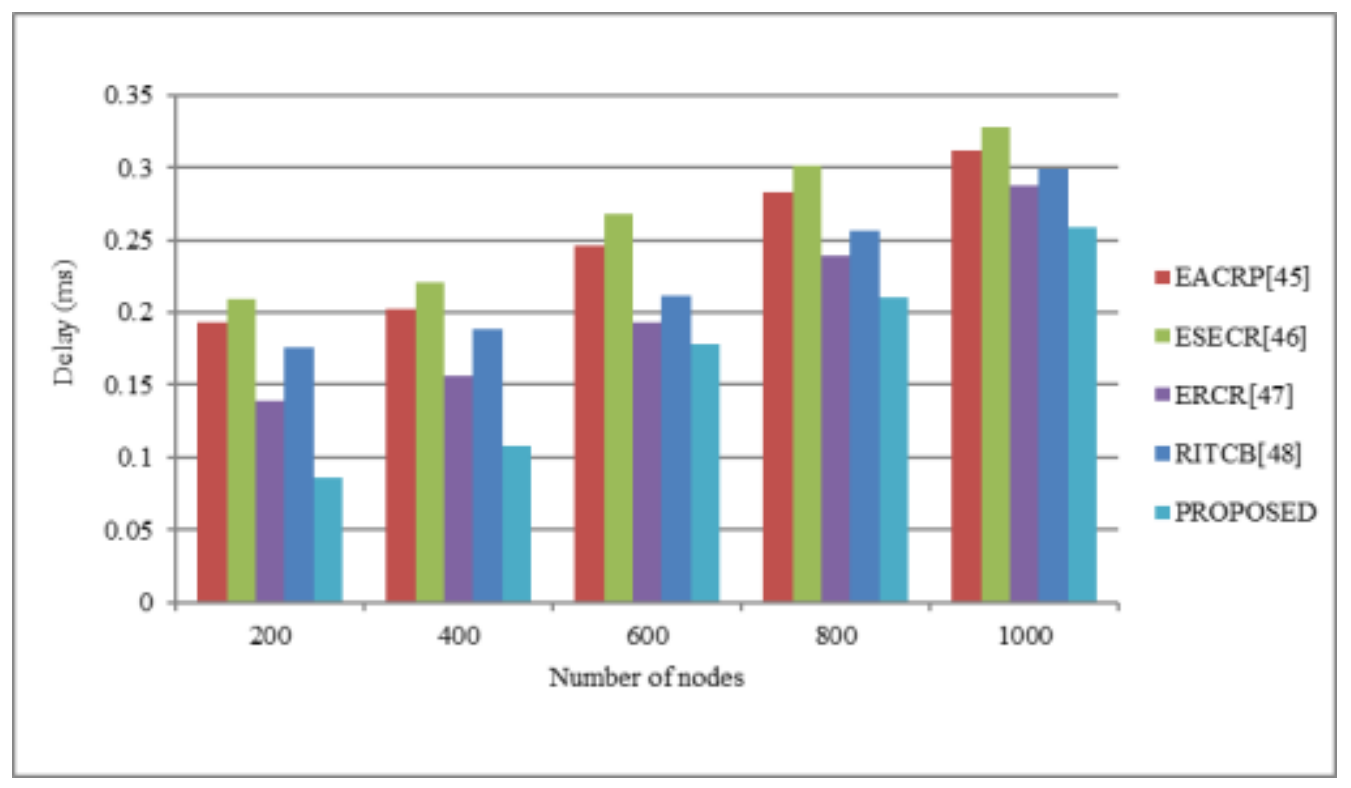

Fig 10: End-to-End Delay

With the assumption of the values presented in Table2, the graph in fig10 is drawn in which the number of the nodes are taken in the $\mathrm{x}$-axis and the delay is taken in the y-axis. These values are obtained from the trace file of the entire network procedure. In figure 10, it demonstrates End-to-End Delay. In this network, the delay is reduced by proposing our work i.e., DFBEER and it performs well will comparing with the existing works like EACRP, ESECR, ERCR, and RITCB. The packets are transmitted through low packet dropping factor links. The low power dissipating nodes reduces ensures quick delivery of data. This reduces the packet drop ratio and drop-free communication links, which helps to reduce the time delay between the data delivery.

Table3: Comparison of different protocols in a throughput performance

\begin{tabular}{|l|l|l|l|l|l|l|l|}
\hline Protocols & 1 & 5 & 10 & 20 & 30 & 40 & 50 \\
\hline EACRP & 212 & 226 & 239 & 258 & 264 & 272 & 289 \\
\hline ESECR & 205 & 219 & 232 & 251 & 256 & 261 & 282 \\
\hline ERCR & 240 & 251 & 259 & 273 & 281 & 290 & 312 \\
\hline RITCB & 222 & 233 & 246 & 267 & 273 & 279 & 296 \\
\hline PROPOSED & 248 & 263 & 270 & 278 & 292 & 301 & 326 \\
\hline
\end{tabular}

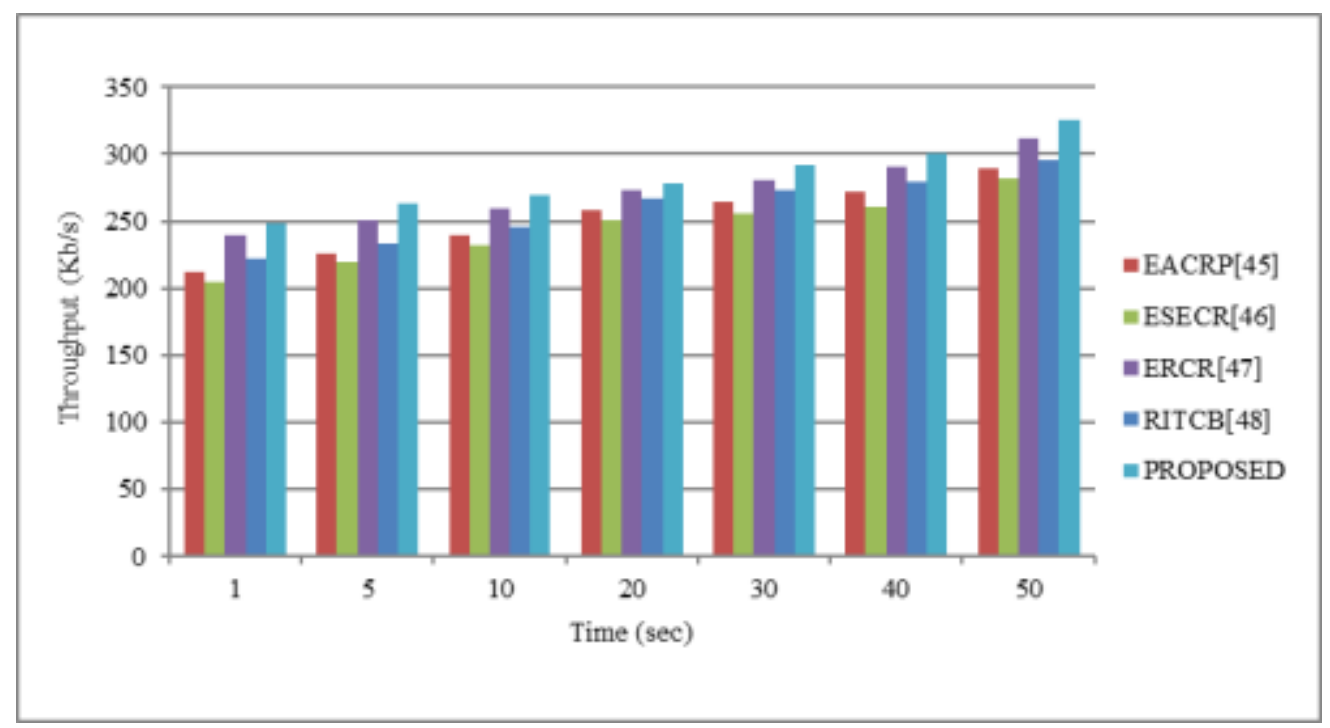

Fig 11: Throughput 
By considering the particulars attained from the trace file of the overall network in Table3, the graph in figure 11 is drawn wherein the $\mathrm{x}$-axis represents the simulation time and $\mathrm{y}$-axis represents the Throughput. In figure 11, it represents the Throughput. The low drop factor \& low power dissipating paths ensure the data delivery rate at the maximum level and ensures additional packets are getting transmitted towards a target node. Within the network, we increased the throughput by proposing our work i.e., DFBEER and it performs well will comparing with the existing works like EACRP, ESECR, ERCR, and RITCB.

Table4: Comparison of different protocols in an energy consumption performance

\begin{tabular}{|l|l|l|l|l|l|}
\hline Protocols & 200 & 400 & 600 & 800 & 1000 \\
\hline EACRP & 8.73 & 9.11 & 11.86 & 15.69 & 18.02 \\
\hline ESECR & 9.26 & 9.87 & 12.22 & 16.11 & 18.89 \\
\hline ERCR & 7.48 & 7.96 & 10.55 & 14.23 & 16.89 \\
\hline RITCB & 8.01 & 8.58 & 11.05 & 14.99 & 17.56 \\
\hline PROPOSED & 6.64 & 7.12 & 9.76 & 11.23 & 13.58 \\
\hline
\end{tabular}

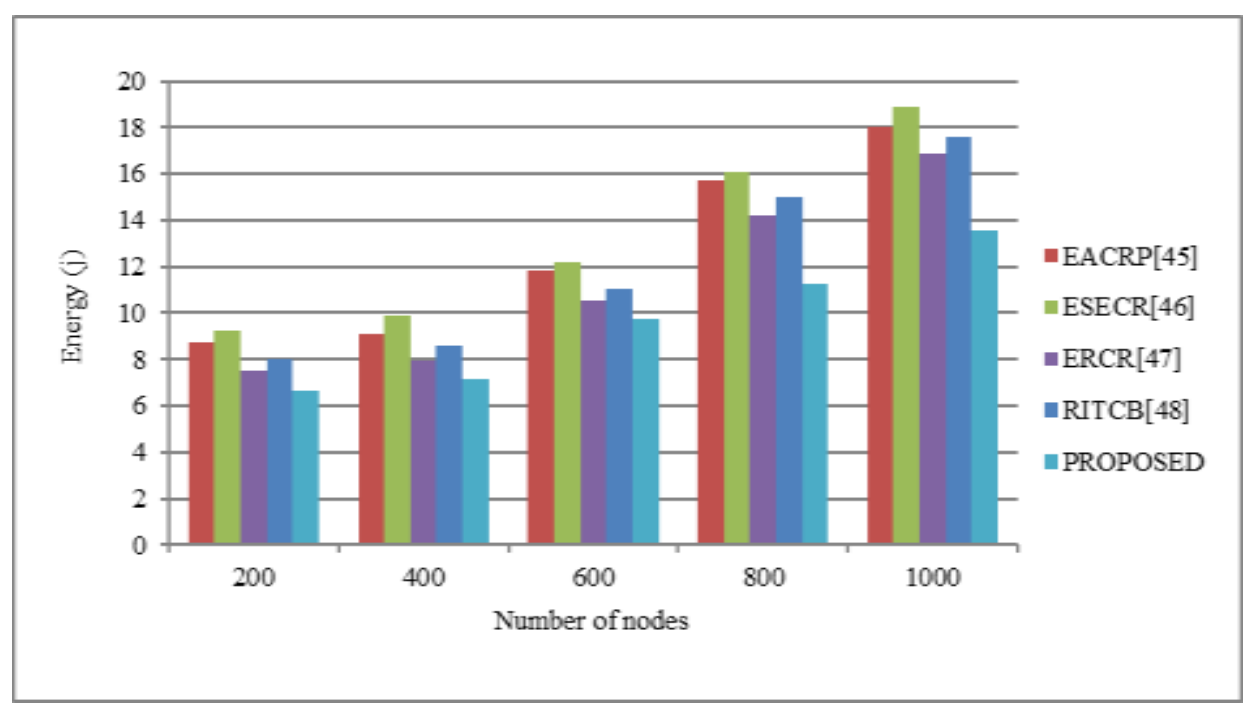

Fig 12: Energy Consumption

With the consideration of the values in Table4, the graph in fig 12 is drawn and the assumption of these values is done from the trace file of the complete network. In the above mentioned graph in figure 12 , the $\mathrm{x}$-axis denotes the number of nodes and the y-axis denotes the Energy. In figure 12, it represents the consumption of energy. Due to the reduced packet drops and the selection of low power dissipation nodes, the rate of retransmission and power wastage is drastically reduced which eliminates unnecessary consumption of energy as well as optimizes energy utilization of a network. Usage of it within the network is decreased by proposing our work i.e., DFBEER and it performs well will comparing with the existing works like EACRP, ESECR, ERCR, and RITCB.

Table 5: Comparison of different protocols in an overhead performance

\begin{tabular}{|l|l|l|l|l|l|}
\hline Protocols & 10 & 20 & 30 & 40 & 50 \\
\hline EACRP & 0.42 & 0.46 & 0.52 & 0.56 & 0.57 \\
\hline ESECR & 0.47 & 0.53 & 0.57 & 0.62 & 0.66 \\
\hline ERCR & 0.38 & 0.4 & 0.43 & 0.46 & 0.49 \\
\hline RITCB & 0.4 & 0.43 & 0.48 & 0.51 & 0.52 \\
\hline PROPOSED & 0.35 & 0.36 & 0.39 & 0.41 & 0.43 \\
\hline
\end{tabular}




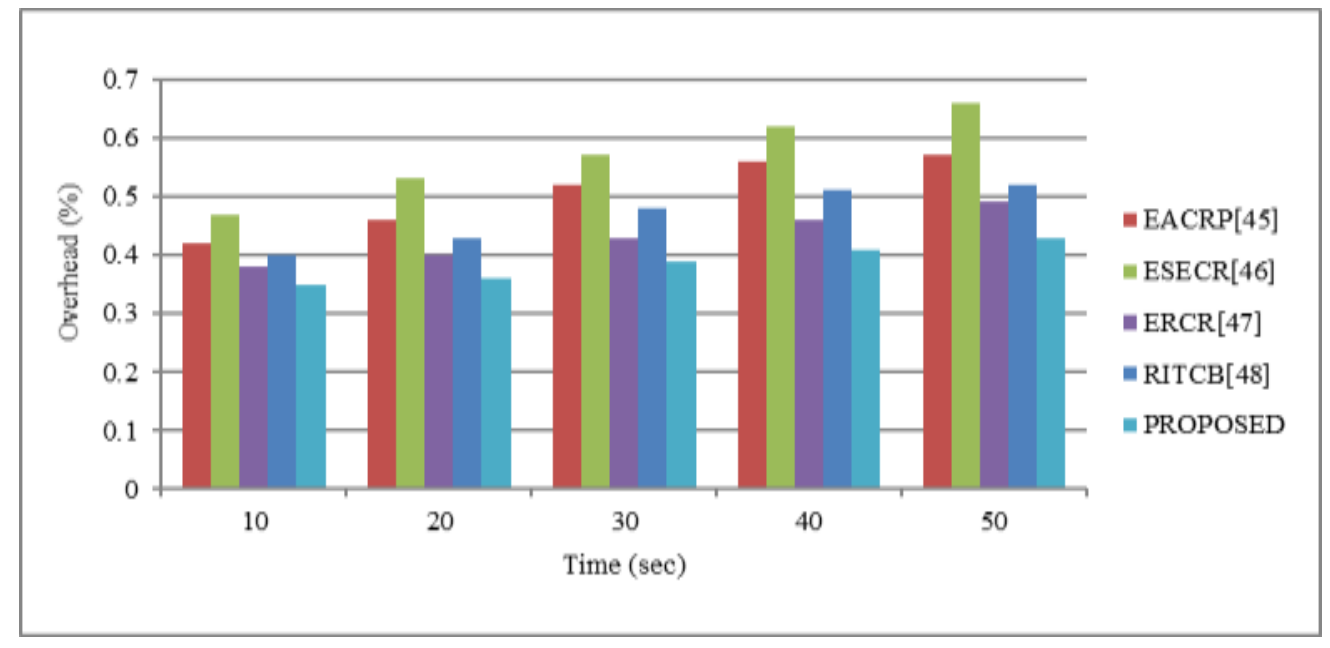

Fig 13: Routing Overhead

The graph in fig 13 is drawn by considering the values achieved from trace file of Table5 in which the simulation time is represented by $\mathrm{x}$-axis and the Overhead is represented by Y-axis. In figure 13, it represents the Routing Overhead. The excessed broadcasting of control packets causes overhead to the network. In our method, the reduced retransmission rate requires only minimum broadcasting of control packets, and low power dissipating nodes require low control packet ratio that decreases the complete overhead of a network. We reduce the routing overhead in the network, by proposing our work i.e., DFBEER and it performs well will comparing with the existing works like EACRP, ESECR, ERCR, and RITCB.

\section{Performance evaluation by traffic protocols:}

This section compares the traffic protocols achieved by proposed algorithm. In order to evaluate the stability of the proposed algorithm under variable traffic conditions and Constant traffic conditions, the VBR traffic source is used with the variable bit rate ranges from 100 to 4400 bytes. CBR traffic source is used which means bit rate is not change for every packet. The parameters like delay, throughput, energy consumption and routing overhead are considered for evaluation.

Table 6: Comparison of different protocols in a delay performance

\begin{tabular}{|l|l|l|l|l|l|}
\hline Traffic values & 200 & 400 & 600 & 800 & 1000 \\
\hline CBR-Proposed & 0.049 & 0.056 & 0.073 & 0.082 & 0.09 \\
\hline VBR-Proposed & 0.071 & 0.079 & 0.089 & 0.097 & 0.102 \\
\hline
\end{tabular}




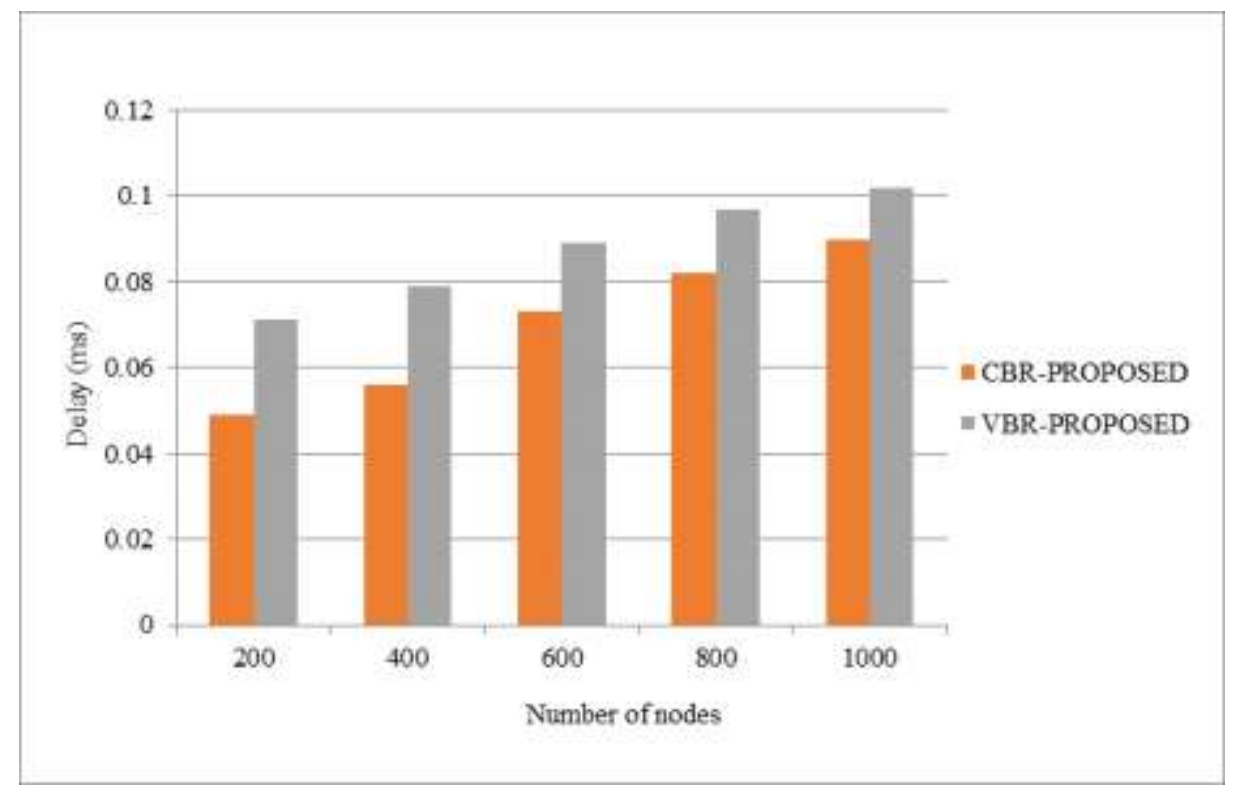

Fig 14: Evaluation of Delay (CBR vs VBR)

With the assumption of the specifications in Table6, the graph in fig 14 is drawn whereas the representation of the number of nodes is done upon x-axis and the representation of the Delay is done upon y-axis. The abovementioned values are obtained from the trace file of the of the whole network procedure. In figure 14, the delay analysis between CBR and VBR traffic protocols for proposed system is represented. The proposed method is tested under constant bit rate CBR in addition to variable bit rate VBR. The results prove that our proposed method performs consistently even in VBR conditions also. There is only a minimum of difference between CBR and VBR values which proves the reliability and stability of the proposed protocol.

Table7: Comparison of different protocols in a throughput performance

\begin{tabular}{|l|l|l|l|l|}
\hline Traffic values & 50 & 100 & 150 & 200 \\
\hline CBR-Proposed & 251 & 256 & 263 & 266 \\
\hline VBR-Proposed & 164 & 172 & 177 & 181 \\
\hline
\end{tabular}

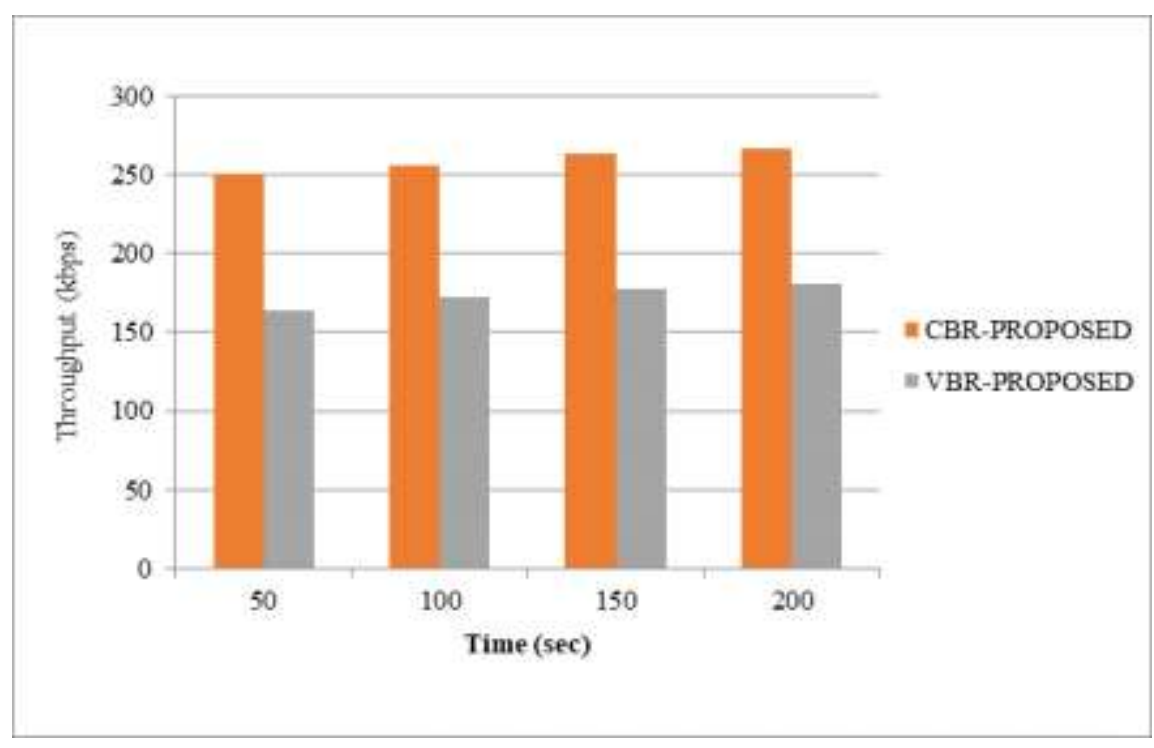

Fig 15: Estimation of Throughput (CBR vs VBR) 
The fig 15 graph is drawn by considering the values out of the trace file of the entire network in the Table 7 . The simulation time is represented upon the $\mathrm{x}$-axis and the Throughput is presented upon the $\mathrm{y}$-axis in the above graph. In fig 15, the throughput analysis between CBR and VBR traffic protocols for proposed system is presented. Usually, throughput ratio gets affected constantly in variable bit rate conditions. But the selection of low drop and low power dissipating nodes maintain the throughput as like as the one in CBR and improves consistently in VBR conditions. It proves that our proposed method ensures reliable data delivery in variable network properties.

Table8: Comparison of different protocols in an energy consumption performance

\begin{tabular}{|l|l|l|l|l|l|}
\hline Traffic values & 200 & 400 & 600 & 800 & 1000 \\
\hline CBR-Proposed & 4.05 & 5.56 & 6.11 & 7.25 & 8.96 \\
\hline VBR-Proposed & 4.9 & 6.02 & 7.1 & 8.06 & 10.11 \\
\hline
\end{tabular}

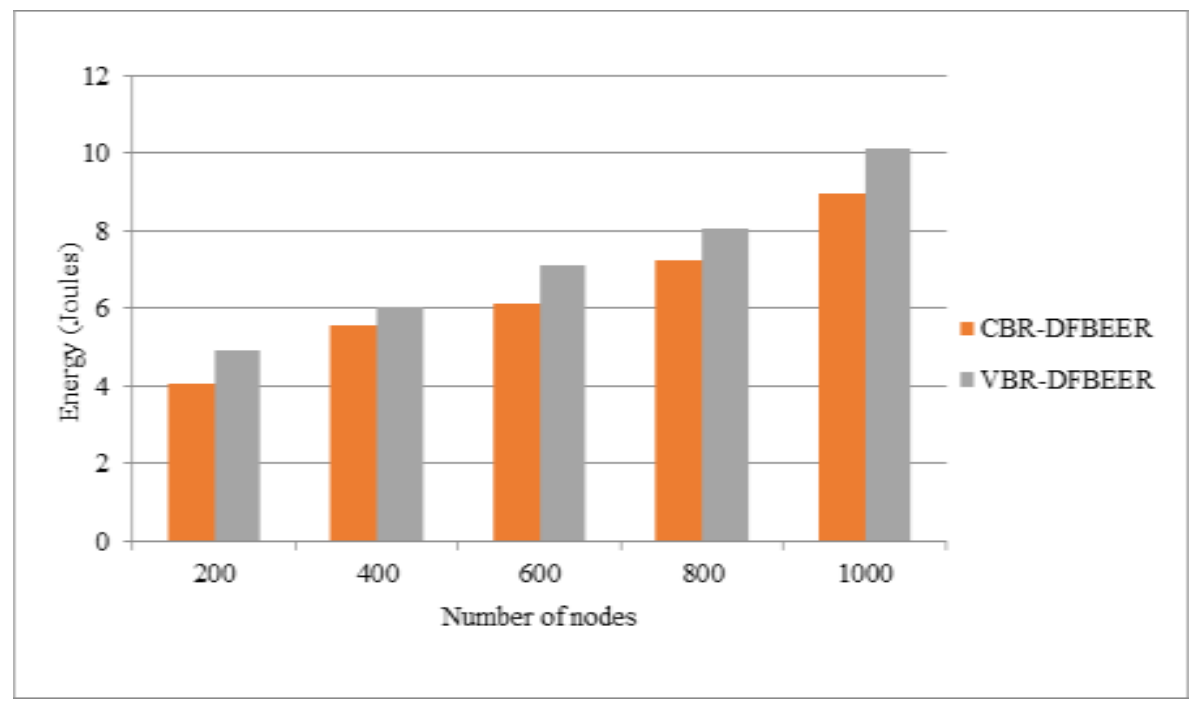

Fig 16: Analysis of Energy consumption (CBR vs VBR)

By assuming the particulars of Table 8 obtained by the trace file of the overall network, the graph in fig 16 is drawn. The performance metrics considered upon the $\mathrm{x}$-axis is the number of nodes and the performance metrics considered upon the y-axis is the Energy. In figure 16, the figure represents the energy consumption analysis between CBR and VBR traffic protocols for the proposed system. The traditional routing algorithms consume high \& irregular power consumption due to the variable bit rate of the transmission. But our proposed protocol maintains a constant behavior in power consumption like CBR in variable conditions.

Table9: Comparison of different protocols in a routing overhead performance

\begin{tabular}{|l|l|l|l|l|}
\hline Traffic values & 50 & 100 & 150 & 200 \\
\hline CBR-Proposed & 0.38 & 0.41 & 0.44 & 0.48 \\
\hline VBR-Proposed & 2.33 & 2.54 & 2.58 & 2.62 \\
\hline
\end{tabular}




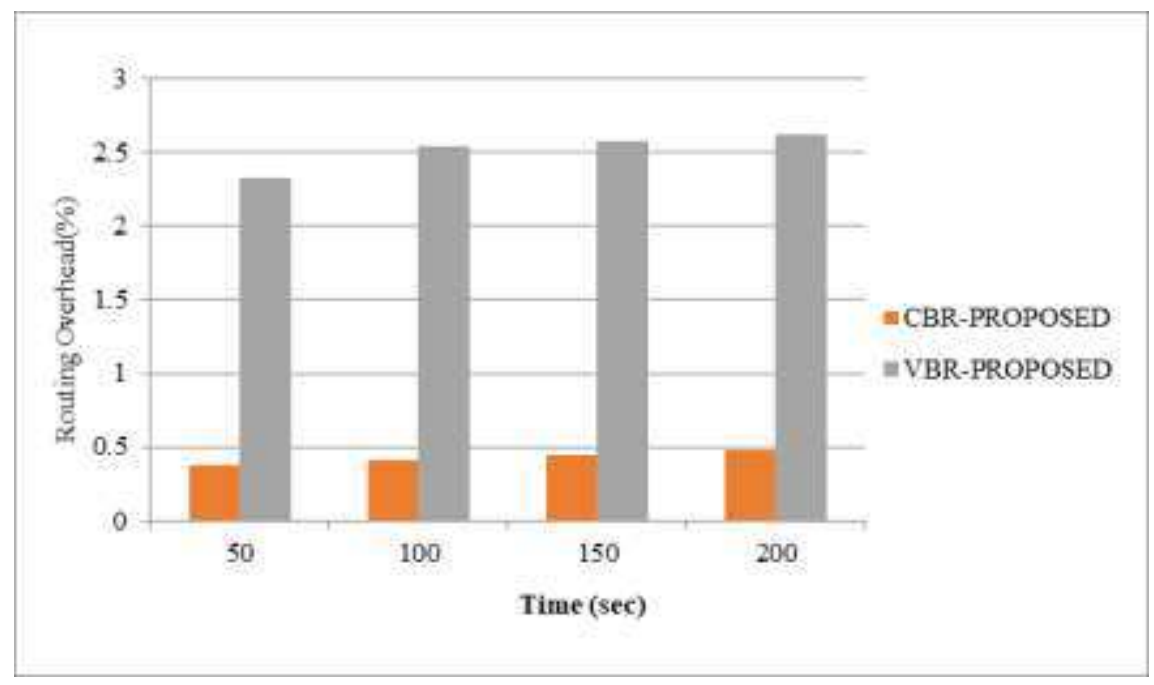

Fig 17: Analysis of Routing Overhead (CBR vs VBR)

The graph in fig 17 is drawn by considering the values in the Table9 in which simulation time is a considered upon $\mathrm{x}$-axis and Routing Overhead is the performance metric assumed on y-axis. The performance metrics are obtained using the trace file of the overall network system. The routing overhead analysis between CBR and VBR traffic protocols for proposed system is represented in figure 17. Usually, overhead ratio gets affected constantly in variable bit rate conditions. But the selection of low drop and low power dissipating nodes maintain the routing overhead as like as the one in CBR and improves consistently in VBR conditions. It proves that our proposed method ensures reliable data delivery in variable network properties.

\section{Conclusion}

In this paper, a novel routing technique is chosen by considering the drop factor of the existing links between the nodes. The drop factor is computed depending upon average hop count of links in addition to the random number generated by each node. The PUs broadcast the control packets over the network and estimate the drop factor of the links. Upon receiving the control packets back, the PU's decides the low drop factor links and checks for the spectrum availability and connects with the target nodes. This will drastically decrease the energy utilization of the network and improves the overall energy consumption of a network. This technique is compared with the existing energy efficiency techniques like EACRP, ESECR, ERCR \& RITCB. The results based on simulation shows that our proposed technique is outperformed all the existing techniques in terms of energy utilization and energy preservation with a better data delivery rate. Also, it is demonstrated by the outcomes that this method minimizes the overall overhead incurred to the network due to these additional algorithms provided to the nodes compared with the existing techniques.

\section{References}

[1] A. El-Mougy, M. Ibnkahla, G. Hattab, and W. Ejaz, Reconfigurable wireless networks, Proceedings of the IEEE 103 (7) (2015) 1125-1158.

[2] W. Ejaz and M. Ibnkahla, Machine-to-machine communications in cognitive cellular systems, in IEEE International Conference on Ubiquitous Wireless Broadband (ICUWB). Montreal, QC: IEEE Oct. (2015)1-5.

[3] M. Ibnkahla, Cooperative Cognitive Radio Networks: The Complete Spectrum Cycle. CRC Press, Taylor \& Francis eBooks (2014).

[4] G. Hattab and M. Ibnkahla, Multiband spectrum access: Great promises for future cognitive radio networks, Proceedings of the IEEE 102 (3) Mar. (2014) 282-306.

[5] W. Ejaz, N. Ul Hasan, and H. S. Kim, Distributed cooperative spectrum sensing in cognitive radio for ad hoc networks, Computer Communications 36 (12) Jul. (2013)1341-1349.

[6] A. El-Mougy and M. Ibnkahla, A cognitive framework for WSN based on weighted cognitive maps and Qlearning, Ad Hoc Networks 16 (2014) 46-69. 
[7] G. A. Shah and O. B. Akan, Spectrum-aware cluster-based routing for cognitive radio sensor networks, in IEEE International Conference on Communications (ICC), IEEE (2013) 2885-2889.

[8] H. Zhang, Z. Zhang, H. Dai, R. Yin, and X. Chen, Distributed spectrum aware clustering in cognitive radio sensor networks, in Global Telecommunications Conference (GLOBECOM), IEEE (2011) 1-6.

[9] W. R. Heinzelman, A. Chandrakasan, and H. Balakrishnan, Energy-efficient communication protocol for wireless microsensor networks, Proceedings of the 33rd annual Hawaii international conference on System Sciences, IEEE 2 (2000) 10 pp.

[10] S. Lindsey and C. S. Raghavendra, Pegasis: Power-efficient gathering in sensor information systems, in Aerospace conference proceedings, IEEE 3 (2002) 3-1125.

[11] A. Manjeshwar and D. P. Agrawal, Apteen, A hybrid protocol for efficient routing and comprehensive information retrieval in wireless sensor networks, in Ipdps 2 (2002) 48.

[12] Y.-F. Wen and W. Liao, On qos routing in wireless ad-hoc cognitive radio networks, in Vehicular Technology Conference (VTC 2010-Spring), IEEE (2010) 1-5.

[13] L. Ding, T. Melodia, S. N. Batalama, J. D. Matyjas, and M. J. Medley, Cross-layer routing and dynamic spectrum allocation in cognitive radio ad hoc networks, IEEE Transactions on Vehicular Technology, IEEE 59 (4) (2010) 1969-1979.

[14] O. Younis and S. Fahmy, Heed: a hybrid, energy-efficient, distributed clustering approach for ad hoc sensor networks, IEEE Transactions on mobile computing, IEEE 3 (4) (2004) 366-379.

[15]. Yick, J., Mukherjee, B., \& Ghosal, D, Wireless sensor network survey. Computer Networks 52(12) (2008) 2292-2330.

[16]. Wang, N., Zhang, N., \& Wang, M., Wireless sensors in agriculture and food industry recent development and future perspective. Computers and Electronics in Agriculture 50(1) (2006) 1-14.

[17]. Qi, L., Xu, M., Fu, Z., Mira, T., \& Zhang, X., C2SLDS: A WSN-based perishable food shelf-life prediction and LSFO strategy decision support system in cold chain logistics. Food Control 38 (2014) 19-29.

[18]. Akyildiz, I. F., Su, W., Sankarasubramaniam, Y., \& Cayirci, E., Wireless sensor networks: A survey. Computer Networks 38(4) (2002) 393-422.

[19]. Bukhari, S. H. R., Siraj, S., \&Rehmani, M. H., Wireless sensor networks in smart cities: Applications of channel bonding to meet data communication requirements. Book Chapter for the Book, Transportation and Power Grid in Smart Cities: Communication Networks and Services. Wiley, UK (in print) (2018) 247-268.

[20]. Liang, Y. C., Zeng, Y., Peh, E. C. Y., \& Hoang, A. T., Sensing-throughput tradeoff for cognitive radio networks. IEEE Transactions on Wireless Communications, IEEE 7(4) (2008) 326-1337.

[21]. Chowdhury, K. R., \& Akyildiz, I. F., Cognitive wireless mesh networks with dynamic spectrum access. IEEE Journal on Selected Areas in Communications, IEEE 26(1) (2008) 168-181.

[22]. Akyildiz, I. F., Lee, W. Y., \& Chowdhury, K. R., CRAHNs: Cognitive radio ad hoc networks. Ad Hoc Networks 7(5) (2009) 810-836.

[23] Mayuri, A.V.R. and Subramanyam, M.V. QoS Variance Aware Spectrum Sensing and Allocation Strategy for Cognitive Radio Wireless Mesh Networks. Global Journal of Computer Science and Technology. 15 (2) (2015).

[24] S. Park, H. Kim, and D. Hong, Cognitive radio networks with energy harvesting, IEEE Transactions on Wireless communications, IEEE 12 (3) (2013) 1386-1397.

[25] H. Gao, W. Ejaz, and M. Jo, Cooperative Wireless Energy Harvesting and Spectrum Sharing in 5G Networks, IEEE Access 4 (2016) 3647-3658.

[26] S. S. Kalamkar, A. Banerjee et al., Energy harvesting cognitive radio with channel-aware sensing strategy, IEEE Communications Letters 18 (7) (2014) 1171-1174.

[27] J.-S. Leu, T.-H. Chiang, M.-C. Yu, and K.-W. Su, "Energy efficient clustering scheme for prolonging the lifetime of wireless sensor network with isolated nodes," IEEE communications letters 19 (2) (2015) $259-262$. 
[28] M. Mishra, C. R. Panigrahi, B. Pati, and J. L. Sarkar, ECHS: An energy aware cluster head selection algorithm in Wireless Sensor Networks, in International Conference on Man and Machine Interfacing (MAMI). Bhubaneswar, India: IEEE (2015) 1-4.

[29] V. Pal, G. Singh, and R. Yadav, Energy efficient cluster head selection scheme: A change in node death scenario of leach for surveillance wireless sensor networks, in IEEE International Conference on Parallel Distributed and Grid Computing (PDGC). Himachal Pradesh, India: IEEE (2012) 865-869.

[30] J. Zhu, C.-H. Lung, and V. Srivastava, A hybrid clustering technique using quantitative and qualitative data for wireless sensor networks, Ad Hoc Networks 25 (2015) 38-53.

[31] B. Pourpeighambar, M. Dehghan, and M. Sabaei, Non-cooperative reinforcement learning based routing in cognitive radio networks, Computer Communications 106 (2017) 11-23.

[32] N. Dutta, H. K. D. Sarma, and Z. Polkowski, Cluster based routing in cognitive radio adhoc networks: Reconnoiteringsinr and ett impact on clustering, Computer Communications 115 (2018) 10-20.

[33] J. Ren, Y. Zhang, Q. Ye, K. Yang, K. Zhang, and X. S. Shen, Exploiting secure and energy-efficient collaborative spectrum sensing for cognitive radio sensor networks, IEEE Transactions on Wireless Communications 15 (10) (2016) 6813-6827.

[34] E. Fadel, M. Faheem, V. C. Gungor, L. Nassef, N. Akkari, M. G. A. Malik, S. Almasri, and I. F. Akyildiz, Spectrum-aware bio-inspired routing in cognitive radio sensor networks for smart grid applications, Computer Communications 101 (2017) 106-120.

[35] J. Abolarinwa, N. M. A. Latiff, and S. K. Syed, Energy-efficient, learning-inspired channel decision and access technique for cognitive radio-based wireless sensor networks, Journal of Multimedia and Ubiquitous Engineering 10 (2) (2015) 11-24.

[36] M. e. a. Tabassum, An energy aware event-driven routing protocol for cognitive radio sensor networks, Wireless Networks 22 (2016) 1523-1536.

[37] C. H. Oey, I. Christian, and S. Moh, Energy-and cognitive-radio-aware routing in cognitive radio sensor networks, International Journal of Distributed Sensor Networks 2012, (2012).

[38]. Wang, C.-W., Wang, L.-C., \& Adachi, F. Modeling and analysis of multi-user spectrum selection schemes in cognitive radio networks. In IEEE 20th international symposium on personal, indoor and mobile radio communications, IEEE (2009) 828-832.

[39]. Li, X., \& Zekavat, S. A. R. Traffic pattern prediction based spectrum sharing for cognitive radios. Den Haag: INTECH Open Access Publisher (2009).

[40]. Song, Y., \& Xie, J. Common hopping based proactive spectrum handoff in cognitive radio ad hoc networks. In Global telecommunications conference (GLOBECOM) IEEE (2010) 1-5.

[41]. Li, X., \&Zekavat, S. A. Traffic pattern prediction and performance investigation for cognitive radio systems. In IEEE wireless communications and networking conference). IEEE (2008) 894-899.

[42]. Yang, L., Cao, L., \& Zheng, H. Proactive channel access in dynamic spectrum networks. Physical Communications Journal 1 (2008) 103-111.

[43]. Liang, Y., \& Wang, J. A flexible delay and energy-efficient routing protocol for cognitive radio network. In 2012 third international conference on intelligent control and information processing, Dalian (2012) 426-430.

[44]. Hou, L., Yeung, K. H., \& Wong, K. Y. SEER: Spectrum and energy-efficient routing protocol for cognitive radio ad hoc networks. Wireless Networks 21(7) (2015) 2357-2368.

[45] Yadav, R.N., Misra, R., \& Saini, D. Energy aware cluster based routing protocol over distributed cognitive radio sensor network. Comput. Commun. 129 (2018) 54-66.

[46] S. Aslam, W. Ejaz and M. Ibnkahla, Energy and Spectral Efficient Cognitive Radio Sensor Networks for Internet of Things, in IEEE Internet of Things Journal 5 (4) (2018) 3220-3233.

[47] Diab, Rita \&Abdrabou, Atef\&Bastaki, Nabil. (2019). An efficient routing protocol for cognitive radio networks of energy-limited devices. Telecommunication Systems. 73 (2020) 577-594. 
[48] Bukhari, Syed \&Rehmani, Mubashir Husain \&Siraj, Sajid. Remaining Idle Time Aware Intelligent Channel Bonding Schemes for Cognitive Radio Sensor Networks. Wireless Networks. 25 (2019) 4523-4539.

[49] A. V. R. Mayuri and M. V. Subramanyam, "MPGA: QOS adequacy latitude aware cooperative spectrum sensing in Cognitive Wireless Mesh Networks by Meticulous Progression based GA," 2015 Conference on Power, Control, Communication and Computational Technologies for Sustainable Growth (PCCCTSG), Kurnool, 2015, pp. 318-325, doi: 10.1109/PCCCTSG.2015.7503937. 
Figures

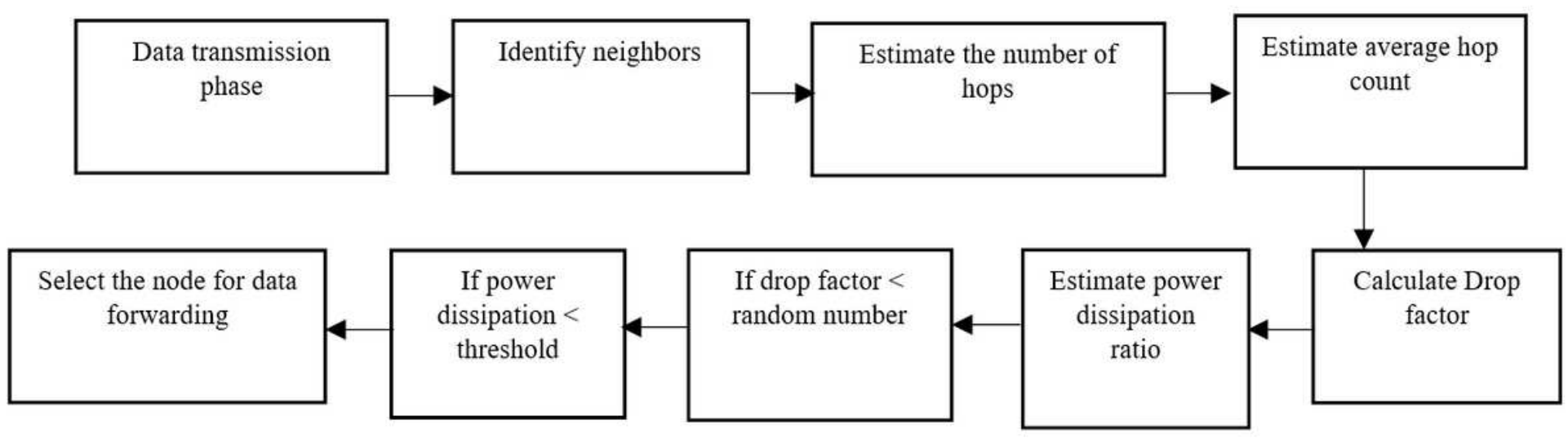

Figure 1

Block diagram of proposed system

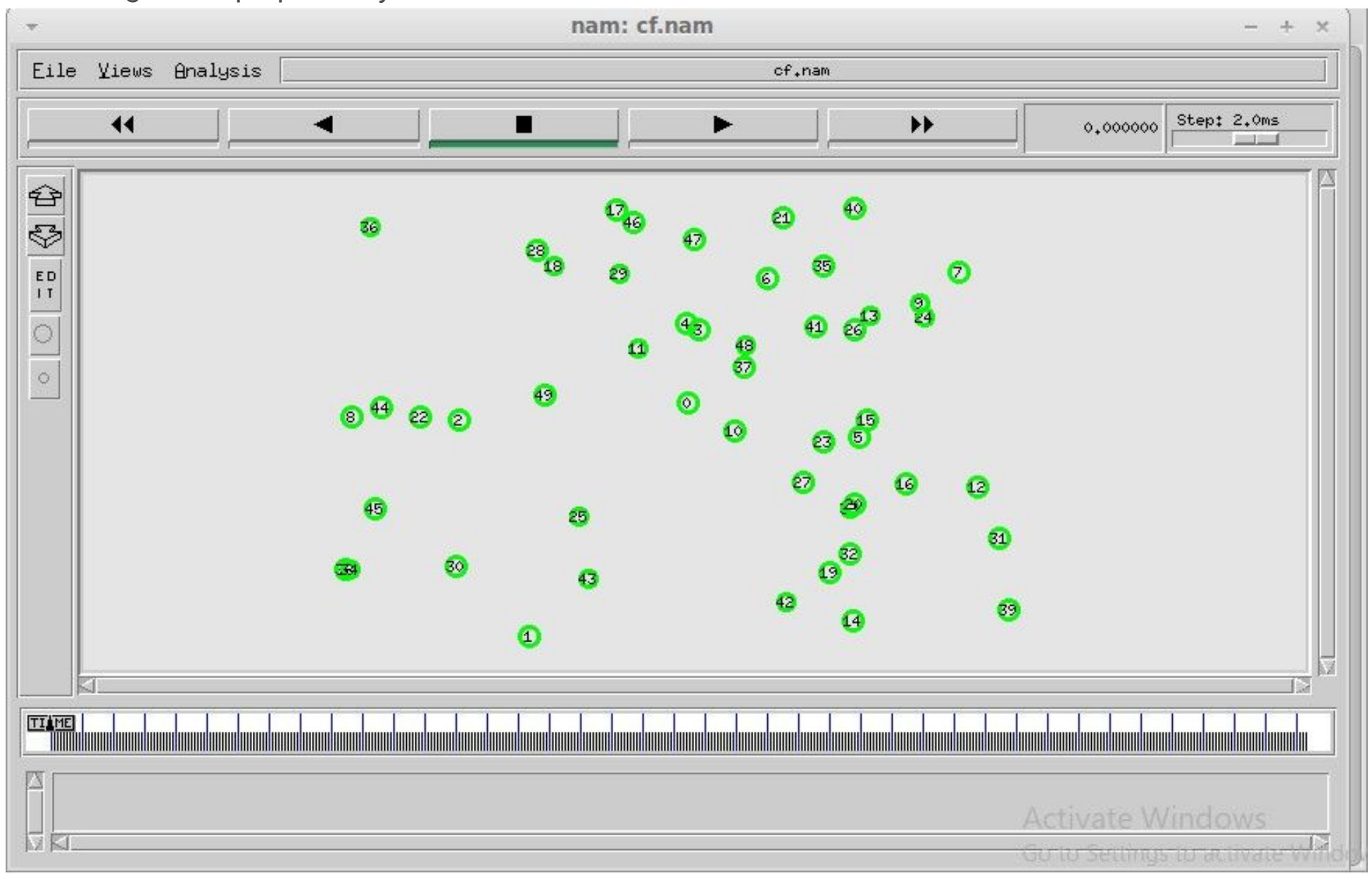

Figure 2

Network Deployment 


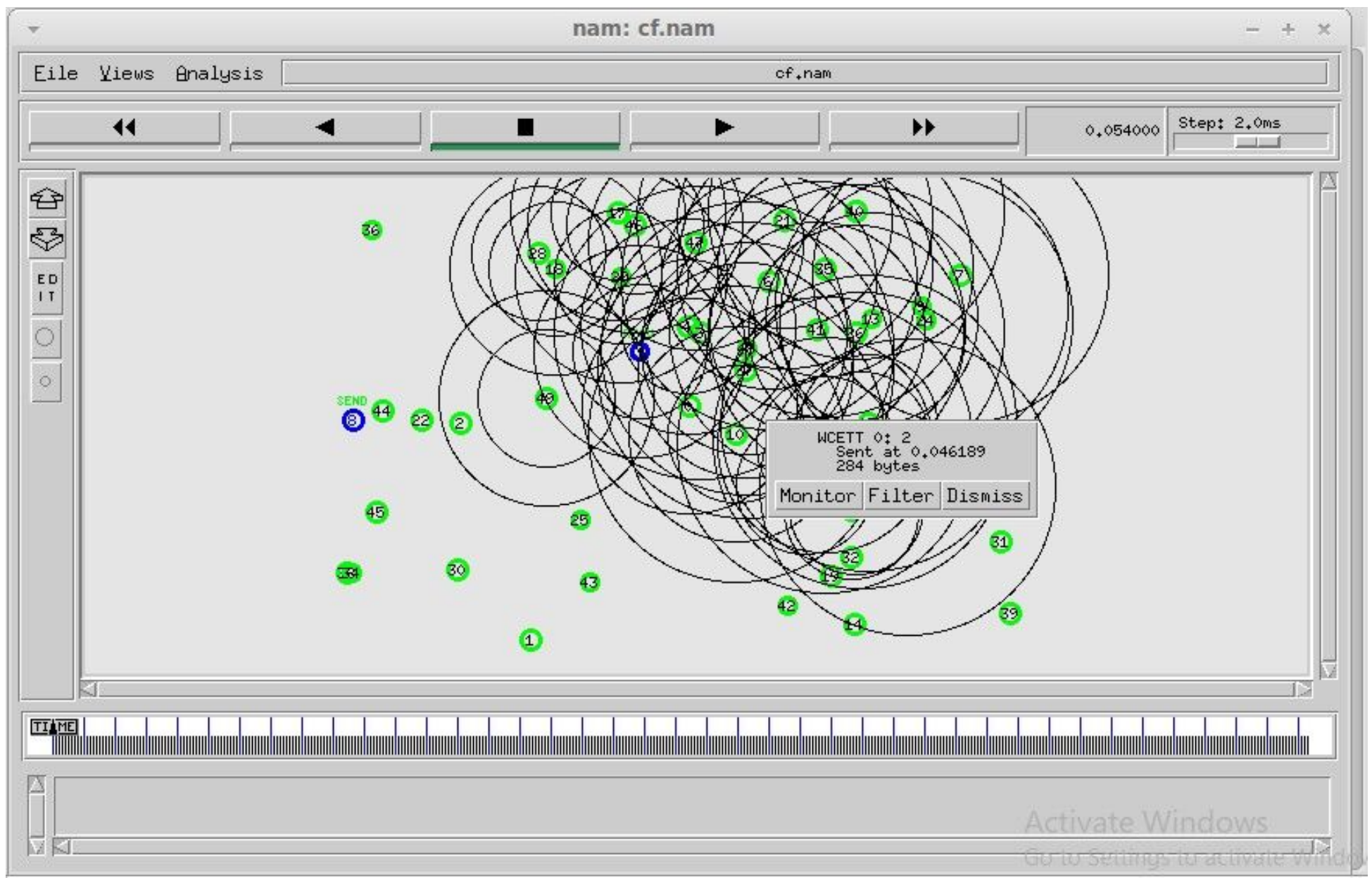

Figure 3

Broadcasting in the network 


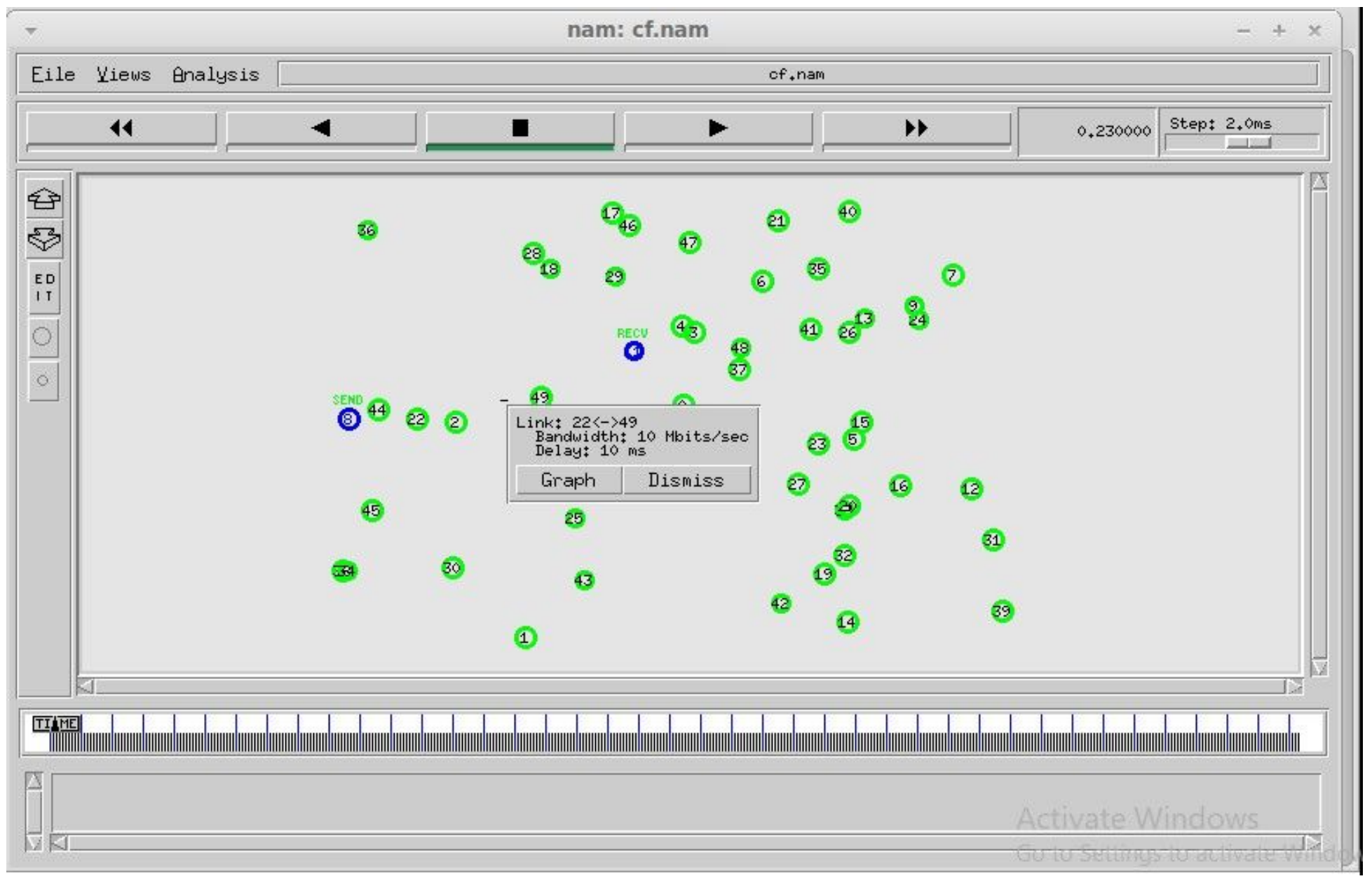

Figure 4

Resource availability in the links 


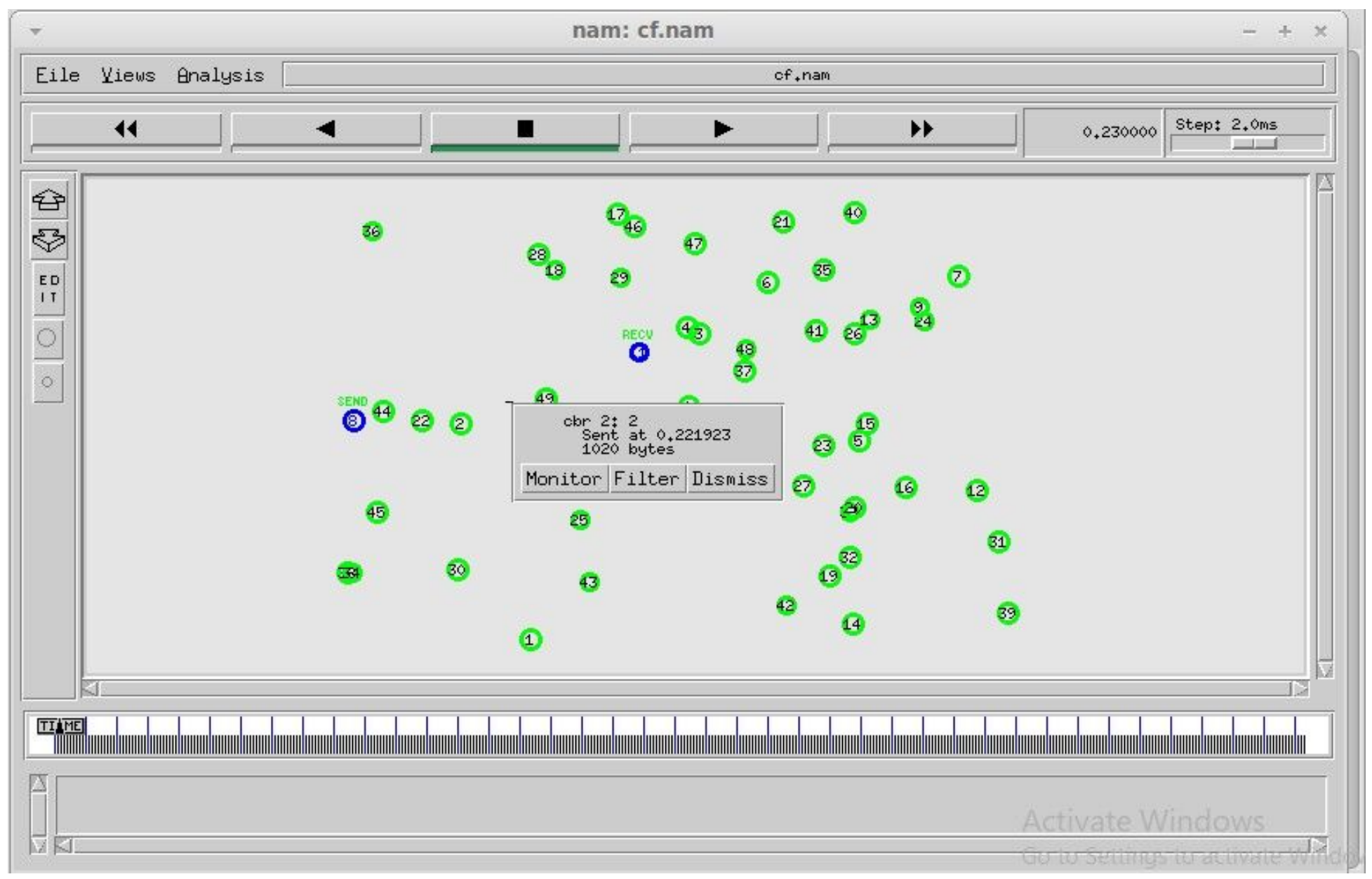

Figure 5

Data transmission at the rate of 1020 bytes between PAIR 1 


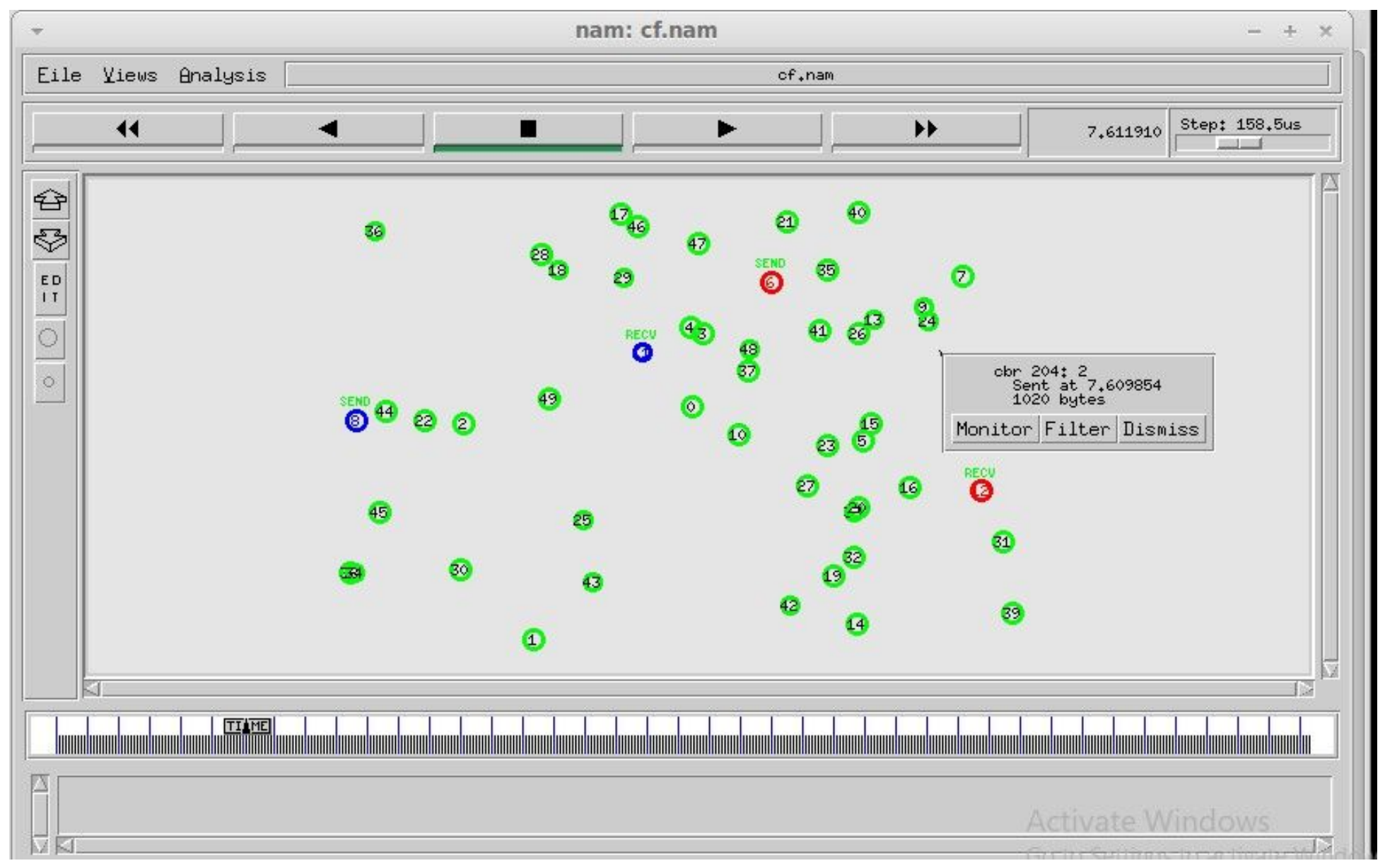

Figure 6

Data transmission at the rate of 1020 bytes between PAIR 2 


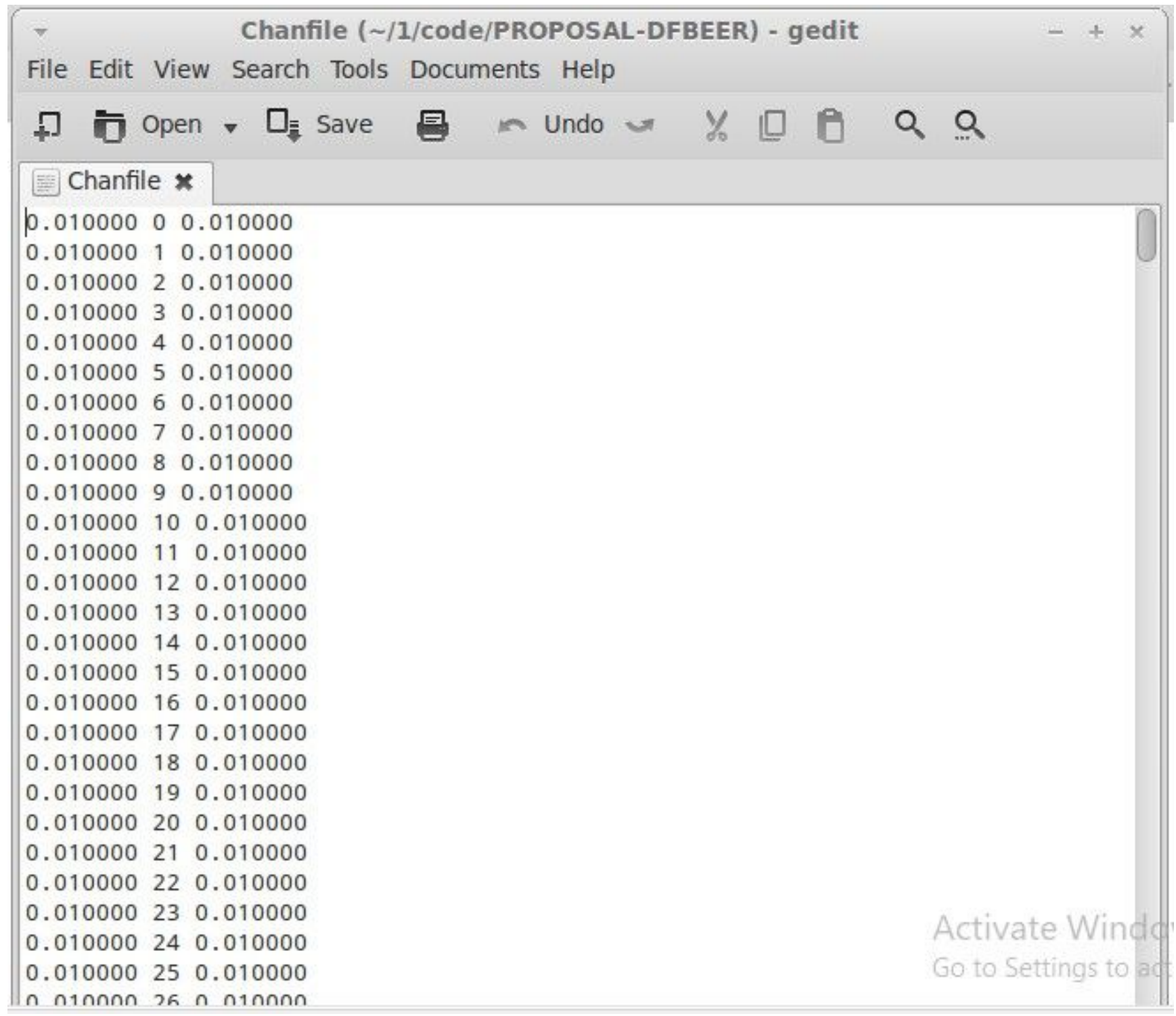

Figure 7

Channel utilization of the nodes during run time (time, node id, utilization) 
File Edit View Search Tools Documents Help

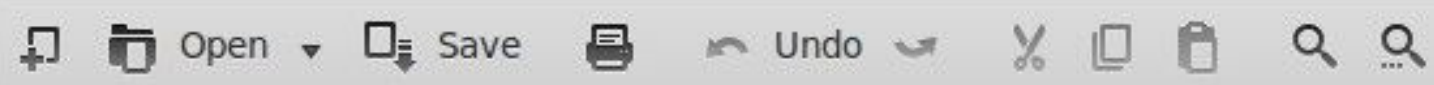

simulation_result.csv $\boldsymbol{*}$

Messages Sent, 1898

Messages Recieved, 1891

Messages Dropped, 7

Drop Rate, 0.00996311907270812

Drop Rate, 0.15128

\section{Figure 8}

Data transmission report after the simulation ends 
File Edit View Search Tools Documents Help

†

Trafficfile $*$

$0.010000 \quad 0 \quad 10$

0.01000015

0.01000027

0.01000038

0.01000048

0.01000056

0.01000066

0.01000076

0.01000086

0.01000096

0.010000108

0.010000119

0.010000123

0.010000136

0.010000145

0.010000156

0.010000165

0.010000174

0.010000188

0.010000195

0.010000207

0.010000216

0.010000226

0.010000236

0.010000246

0.010000258

0.010000266

0.010000277

Figure 9

Traffic report of the nodes during run time (time, node id, traffic count) 


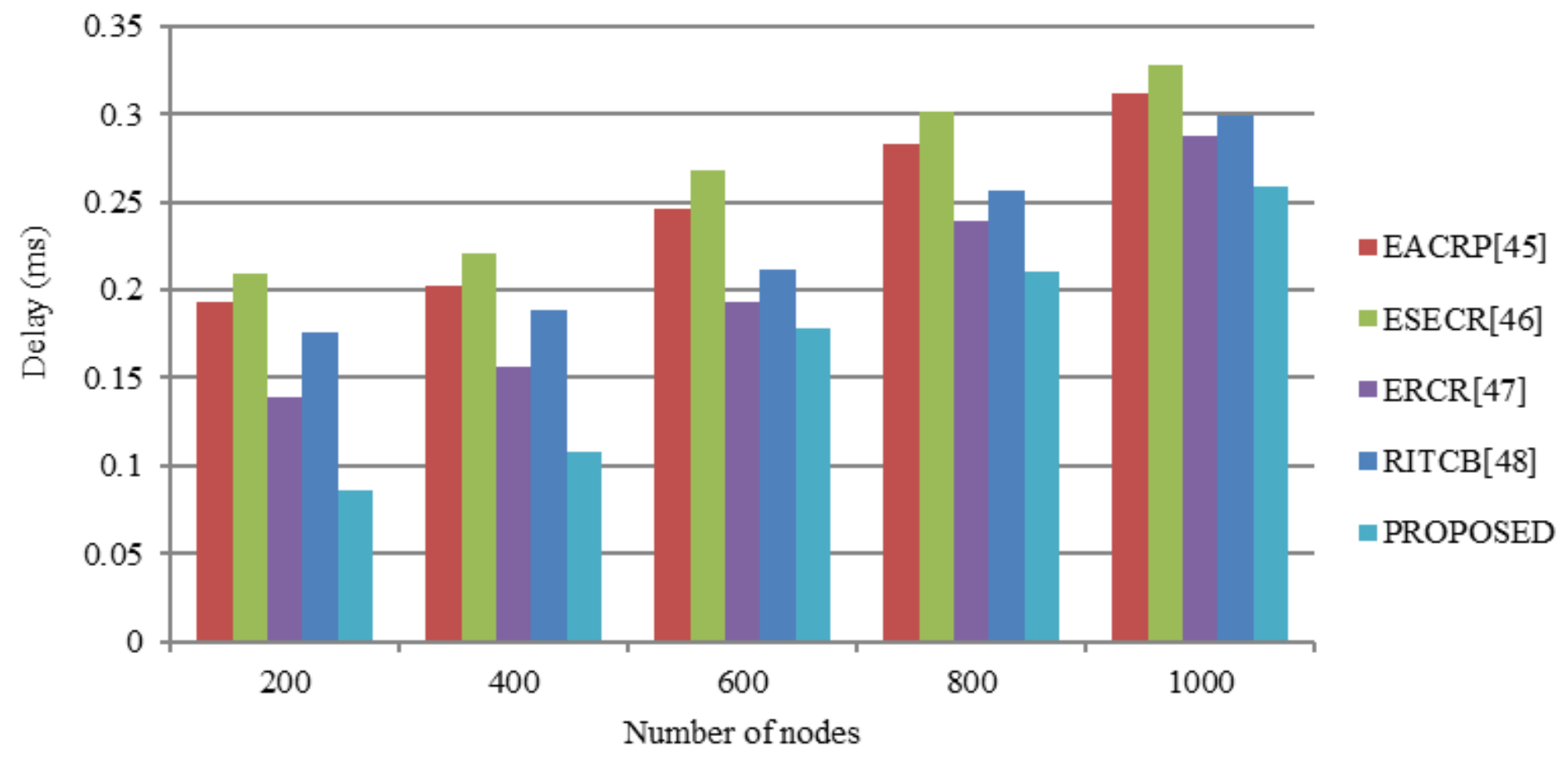

Figure 10

End-to-End Delay

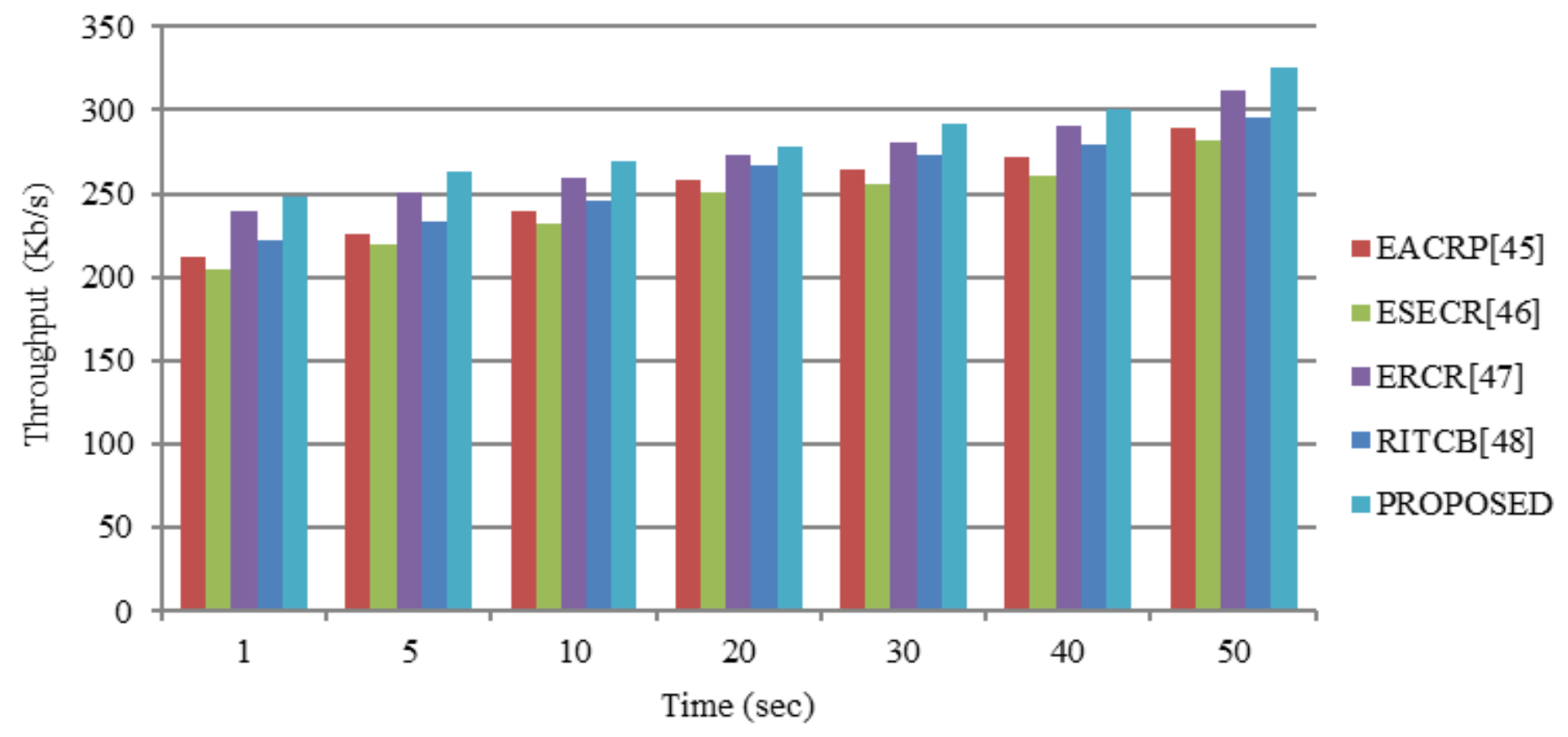

Figure 11 


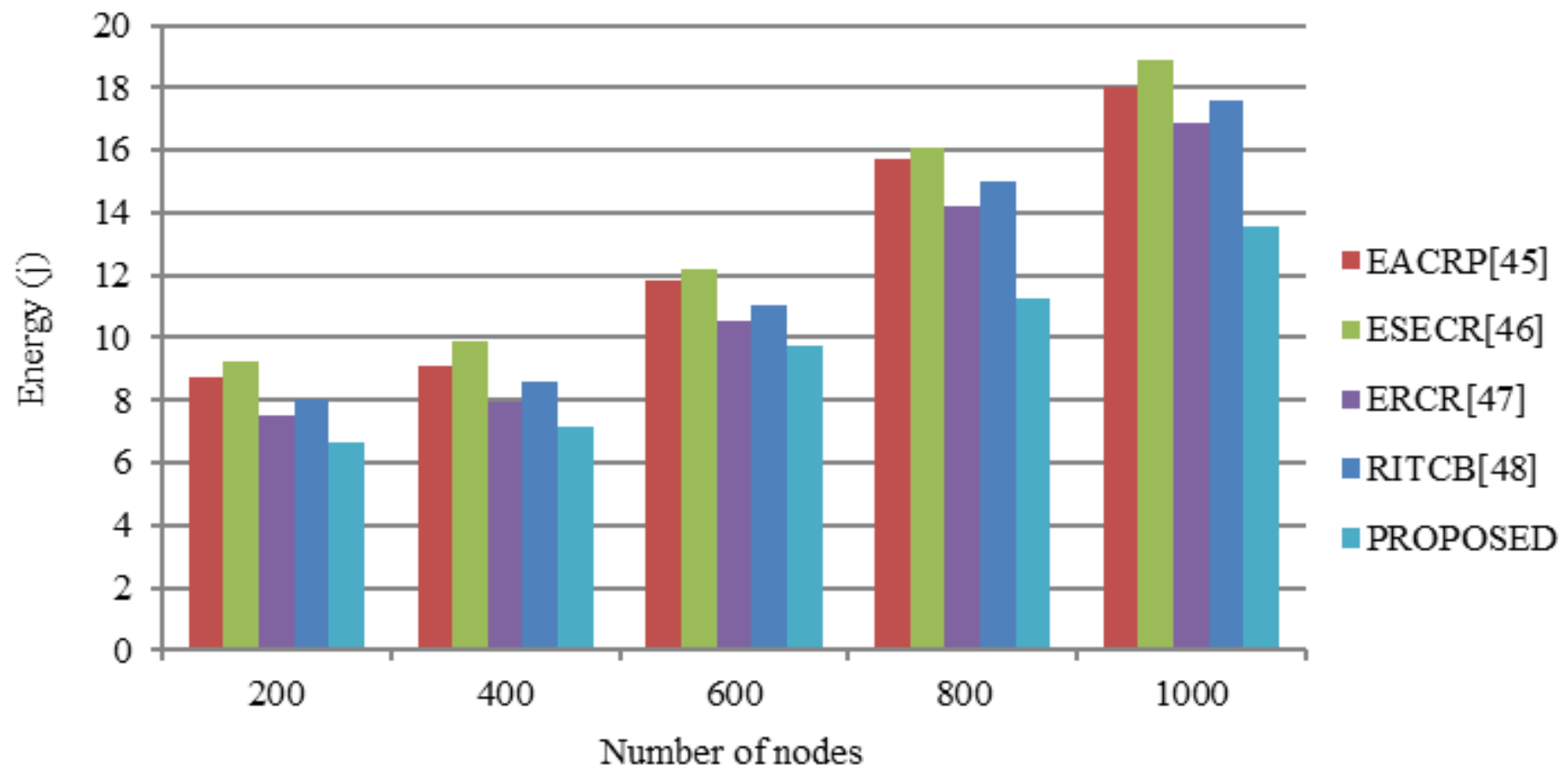

Figure 12

Energy Consumption

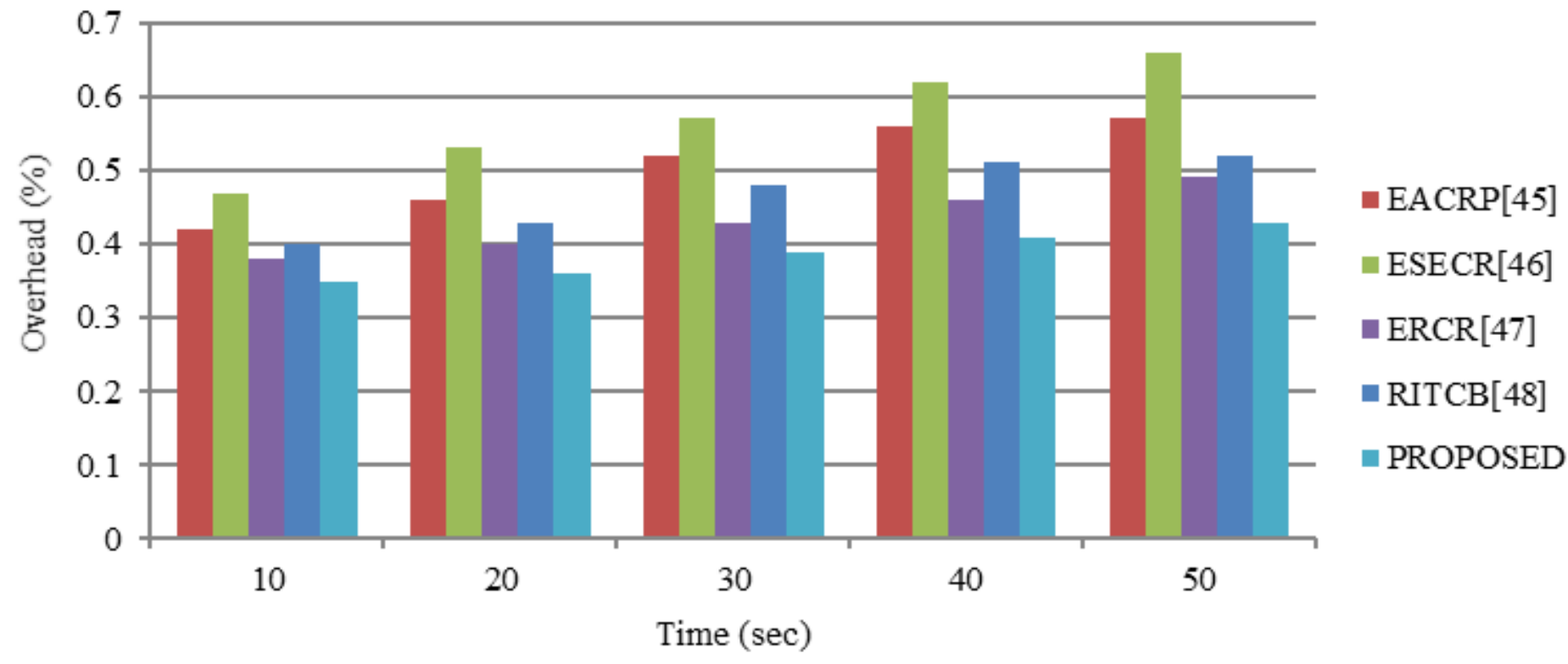

Figure 13 
Routing Overhead

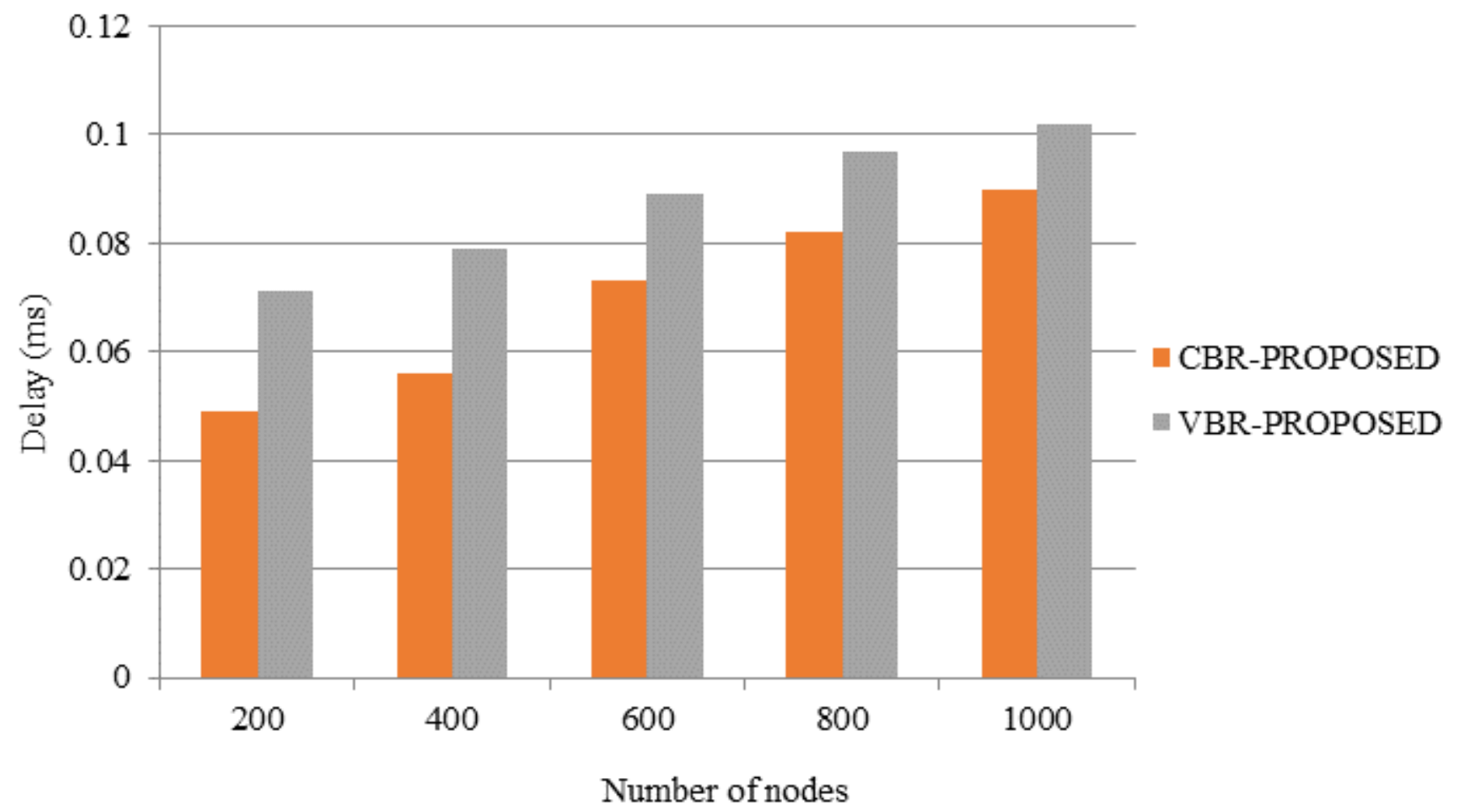

Figure 14

Evaluation of Delay (CBR vs VBR) 


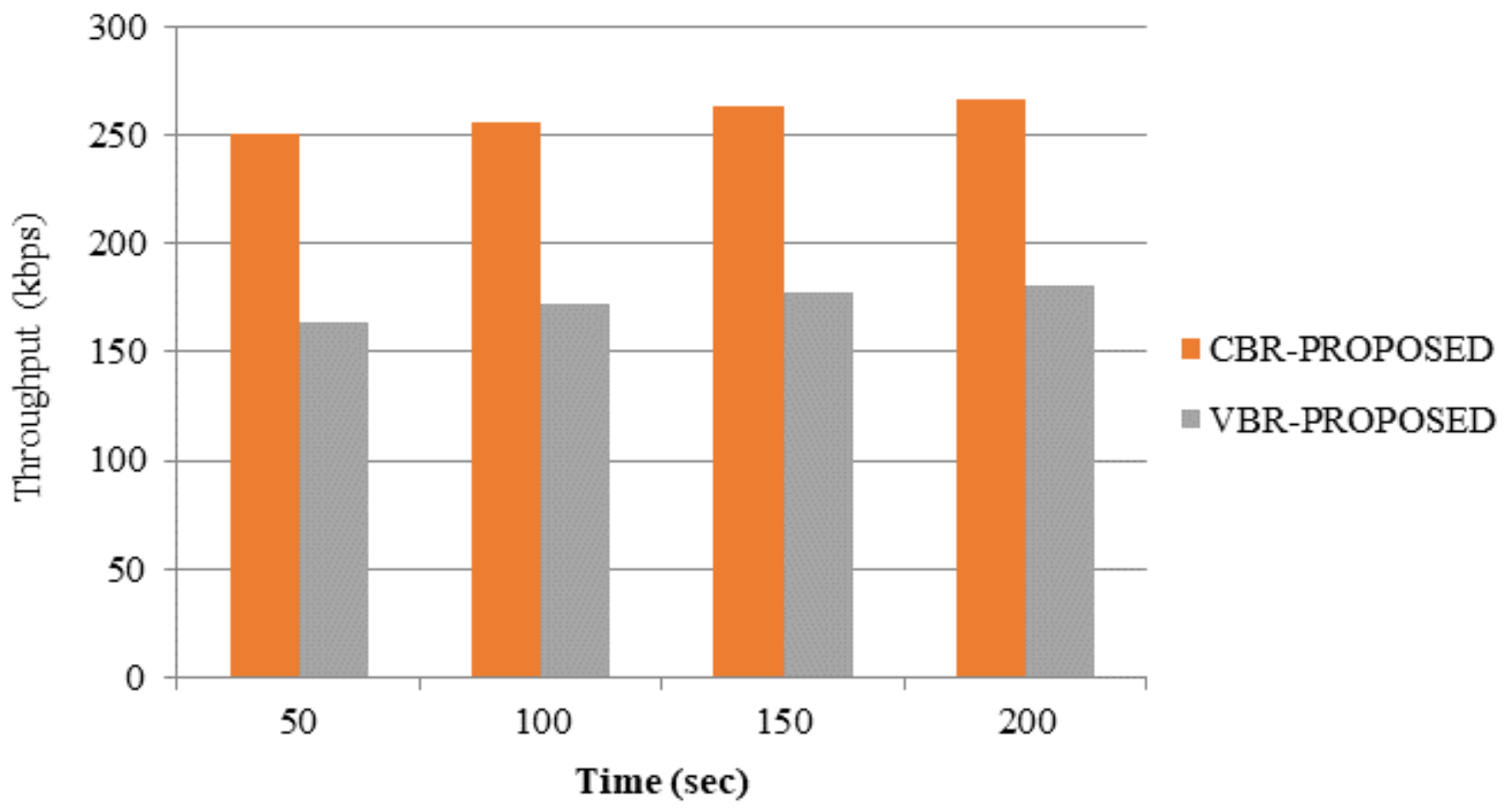

Figure 15

Estimation of Throughput (CBR vs VBR)

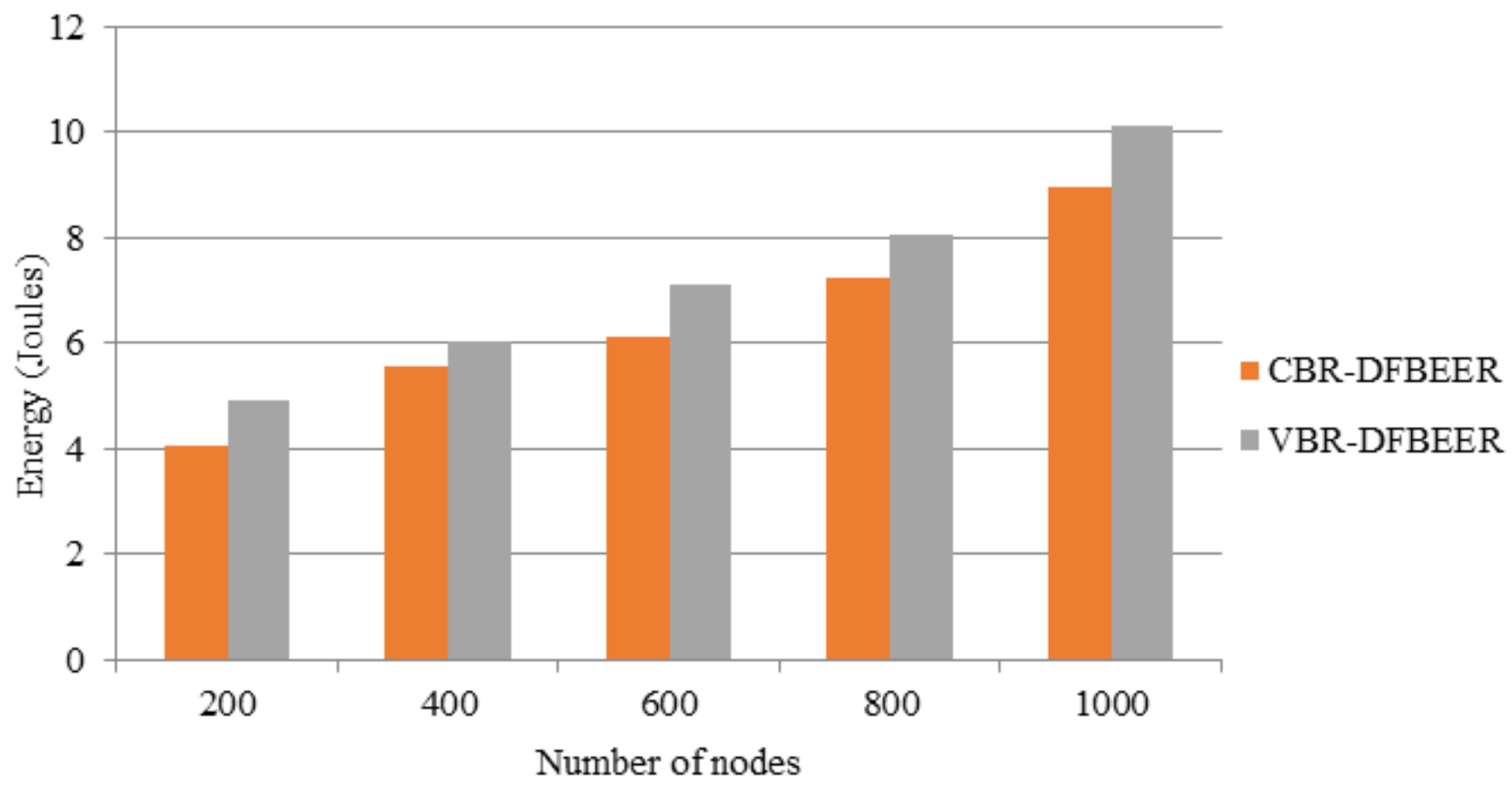


Figure 16

Analysis of Energy consumption (CBR vs VBR)

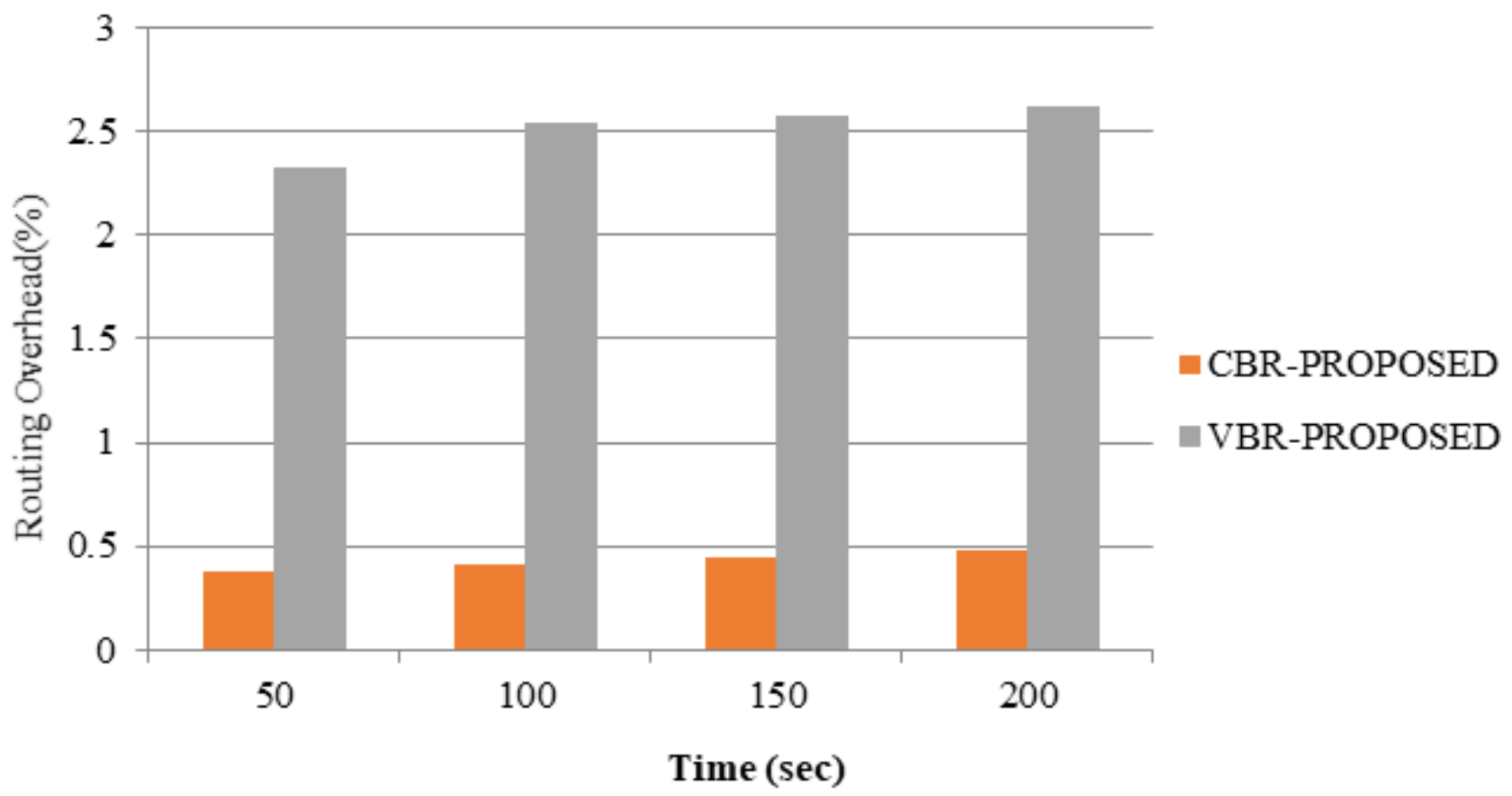

Figure 17

Analysis of Routing Overhead (CBR vs VBR) 